Article

\title{
Geochronology and Geochemistry of Uraninite and Coffinite: Insights into Ore-Forming Process in the Pegmatite-Hosted Uraniferous Province, North Qinling, Central China
}

\author{
Feng Yuan ${ }^{1}$, Shao-Yong Jiang ${ }^{1, *}$, Jiajun Liu ${ }^{2, *}$, Shuai Zhang ${ }^{2}$, Zhibin Xiao ${ }^{3}$, Gang Liu ${ }^{2}$ and \\ Xiaojia $\mathrm{Hu}^{4}$ \\ 1 State Key Laboratory of Geological Processes and Mineral Resources, Collaborative Innovation Centre for \\ Exploration of Strategic Mineral Resources, School of Earth Resources, China University of Geosciences, \\ Wuhan 430074, China; yuanfengyf666@163.com \\ 2 State Key Laboratory of Geological Processes and Mineral Resources, School of Earth Sciences, \\ China University of Geosciences, Beijing 100083, China; zhangshuai@cugb.edu.cn (S.Z.); \\ 18782959317@163.com (G.L.) \\ 3 Tianjin Center, China Geological Survey, Tianjin 300170, China; luqingxzb@163.com \\ 4 Geological Team 224, Shaanxi Nuclear Industry Geological Bureau, Xi'an 710000, China; \\ huxiaojia_123@126.com \\ * Correspondence: shyjiang@cug.edu.cn (S.-Y.J.); liujiajun@cugb.edu.cn (J.L.); Tel.: +027-67883058 (S.-Y.J.)
}

Received: 5 July 2019; Accepted: 2 September 2019; Published: 13 September 2019

\begin{abstract}
The biotite pegmatites in the Shangdan domain of the North Qinling orogenic belt contain economic concentrations of $U$, constituting a low-grade, large-tonnage pegmatite-hosted uraniferous province. Uraninite is predominant and ubiquitous ore mineral and coffinite is common alteration mineral after initial deposit formation. A comprehensive survey of the uraninite and coffinite assemblage of the Chenjiazhuang, Xiaohuacha, and Guangshigou biotite pegmatites in this uraniferous province reveal the primary magmatic $U$ mineralization and its response during subsequent hydrothermal events. Integrating the ID-TIMS (Isotope Dilution Thermal Ionization Mass Spectrometry) ${ }^{206} \mathrm{~Pb} /{ }^{238} \mathrm{U}$ ages and U-Th- $\mathrm{Pb}$ chemical ages for the uraninites with those reported from previous studies suggests that the timing of $U$ mineralization in the uraniferous province was constrained at 407-415 Ma, confirming an Early Devonian magmatic ore-forming event. Based on microtextural relationships and compositional variation, three generations of uranium minerals can be identified: uaninite-A (high Th-low U-variable Y group), uranite-B (low Th-high U, Y group), and coffinite (high $\mathrm{Si}$, Ca-low $\mathrm{U}, \mathrm{Pb}$ group). Petrographic and microanalytical observations support a three-stage evolution model of uranium minerals from primary to subsequent generations as follows: (1) during the Early Devonian (stage 1), U derived from the hydrous silicate melt was mainly concentrated in primary magmatic uaninite-A by high- $\mathrm{T}\left(450-607^{\circ} \mathrm{C}\right)$ precipitation; (2) during the Late Devonian (stage 2), U was mobilized and dissolved from pre-existing uraninite-A by U-bearing fluids and in situ reprecipitated as uraninite- $\mathrm{B}$ under reduced conditions. The in situ transformation of primary uraninite-A to second uraninite-B represent a local medium- $\mathrm{T}\left(250-450^{\circ} \mathrm{C}\right)$ hydrothermal U-event; and (3) during the later low- $\mathrm{T}\left(100-140{ }^{\circ} \mathrm{C}\right)$ hydrothermal alteration (stage 3), $\mathrm{U}$ was remobilized and derived from the dissolution of pre-existing uraninite by $\mathrm{CO}_{2}$ - and $\mathrm{SiO}_{2}$-rich fluids and interacted with reducing agent (e.g., pyrite) leading to reprecipitation of coffinite. This process represents a regional and extensive low-T hydrothermal U-event. In view of this, $\mathrm{U}$ minerals evolved from magmatic uraninite-A though fluid-induced recrystallized uraninite-B to coffinite, revealing three episodes of $U$ circulation in the magmatic-hydrothermal system.
\end{abstract}


Keywords: uraninite; coffinite; U circulation; ID-TIMS; chemical composition; Shangdan pegmatite-hosted uraniferous province

\section{Introduction}

There is an increased demand for $U$ resources, referred to as strategic metal resources and clean energy, with development of nuclear reactors for electric power generation [1]. Renewed interest in biotite pegmatites has arisen due to their economic $U$ endowment [2-5]. The most important $U$ hosts in pegmatite-type $U$ deposits are uraninite and coffinite, common minerals containing reduced $\mathrm{U}^{4+}$ found in nature [6-8]. Self-oxidation caused by radioactive decay of $U$, and valence compensation due to prevalent cationic substitutions have collectively contributed to the coexistence of mixed $\mathrm{U}^{4+}$ and $\mathrm{U}^{6+}$ in uraninite and coffinite structure $[9,10]$. Thus, nonstoichiometric $\mathrm{UO}_{2}+\mathrm{x}$ compound with a fluorite $\left(\mathrm{CaF}_{2}\right)$-type structure of uraninite and $\mathrm{U}\left(\mathrm{SiO}_{4}\right)_{1-\mathrm{x}}(\mathrm{OH})_{\mathrm{x}}$ with zircon $\left(\mathrm{ZrSiO}_{4}\right)$ - or thorite $\left(\mathrm{ThSiO}_{4}\right)$-type structure of coffinite prevalently occur in nature [8].

Numerous studies have shown that uraninite and coffinite have the ability to retain compositional zoning through time and varying physico-chemical conditions, and thus can track an evolution of $\mathrm{U}$ in the magmatic-hydrothermal systems [9-14]. For pegmatite-type deposits, uraninite is mainly concentrated by magmatic process, but being one of the most sensitive mineral phases to post-magmatic hydrothermal processes due to geochemical instability to readily dissolve in the presence of later oxidizing fluids [15-17]. Uraninite is capable of retaining the imprints of the earlier magmatic event and capturing the footprints of subsequent hydrothermal events [18,19]. On the other hand, recent researches suggest that mobilized $U$ can be subsequently reprecipitated as coffinite during later silica-rich fluid circulation events [10,20]. Accordingly, the evolution of $U$ mineral generations is instructive for understanding $U$ circulation from magmatic to hydrothermal stages during pegmatite development.

The largest known major accumulations of pegmatite-hosted $U$ in China are $U$ minerals concentrated in the biotite pegmatites from the Shangdan uraniferous province. Intensive efforts by previous studies have been undertaken to investigate the geology, magma origin, petrogenesis, and mineralization mechanism of individual uraniferous pegmatites (e.g., [21,22]). However, comparatively few studies on the regional uraniferous pegmatites as a whole have expounded the circulation from magmatic mineralization to hydrothermal mobility of $U$, which is crucial to understanding the entire mineralization history of the uraniferous province.

In the present study, we investigate the in situ chemical composition of uraninite and coffinite, as well as employing U-Pb ID-TIMS and EPMA chemical U-Th-Pb dating of uraninite. Integrating petrographic examination, geochronology, and mineral chemical data of uraninite and coffinite, we aim to (1) directly constrain the timing of U mineralization using ore mineral; (2) chemically characterize uraninite and coffinite; and (3) provide a detailed reconstruction of the magmatic-hydrothermal circulation of $U$ in the pegmatitic system.

\section{Geological Background}

The Qinling Orogen, being the western part of the Qinling-Dabie-Sulu Orogen in China, is located between the North China Block (NCB) to the north and South China Block (SCB) to the south (Figure 1a,b, [23,24]). It is segmented into four terrains by Luonan-Luanchuan fault (LLF), Shangdan suture zone (SDSZ), and Mianlue suture zone respectively, including the southern margin of the NCB, North Qinling orogenic belt (NQB), South Qinling orogenic belt (SQB), and northern margin of the SCB in a sequence from the north to the source $[25,26]$. The SDSZ, characterized by ophiolitic mélanges and arc-related volcanic rocks, marks the boundary between the NQB and SQB, which is suggested to be formed by the northward subduction of the Shangdan Oceanic lithosphere beneath the NQB continental lithosphere during the Late Cambrian to Ordovician [27,28]. The SDSZ then experienced intra-plate strike-slip faulting during the Mesozoic-Cenozoic [29]. 
The NQB is situated between the northern LLF (F1 in Figure 1c) and southern SDSZ (Figure 1c), which is compatible with a thrusting style of deformation, imbricated by thick-skinned southward-verging thrusts and folds [30]. Pioneering studies have been conducted on the tectonic affinity of the NQB, but there remains considerable debate. Two competing hypotheses have been proposed, involving (I) a separation from the SCB during the middle Neoproterozoic Rodinian supercontinent breakup (e.g., [31,32]); and (II) an independent micro-block (e.g., [26]).
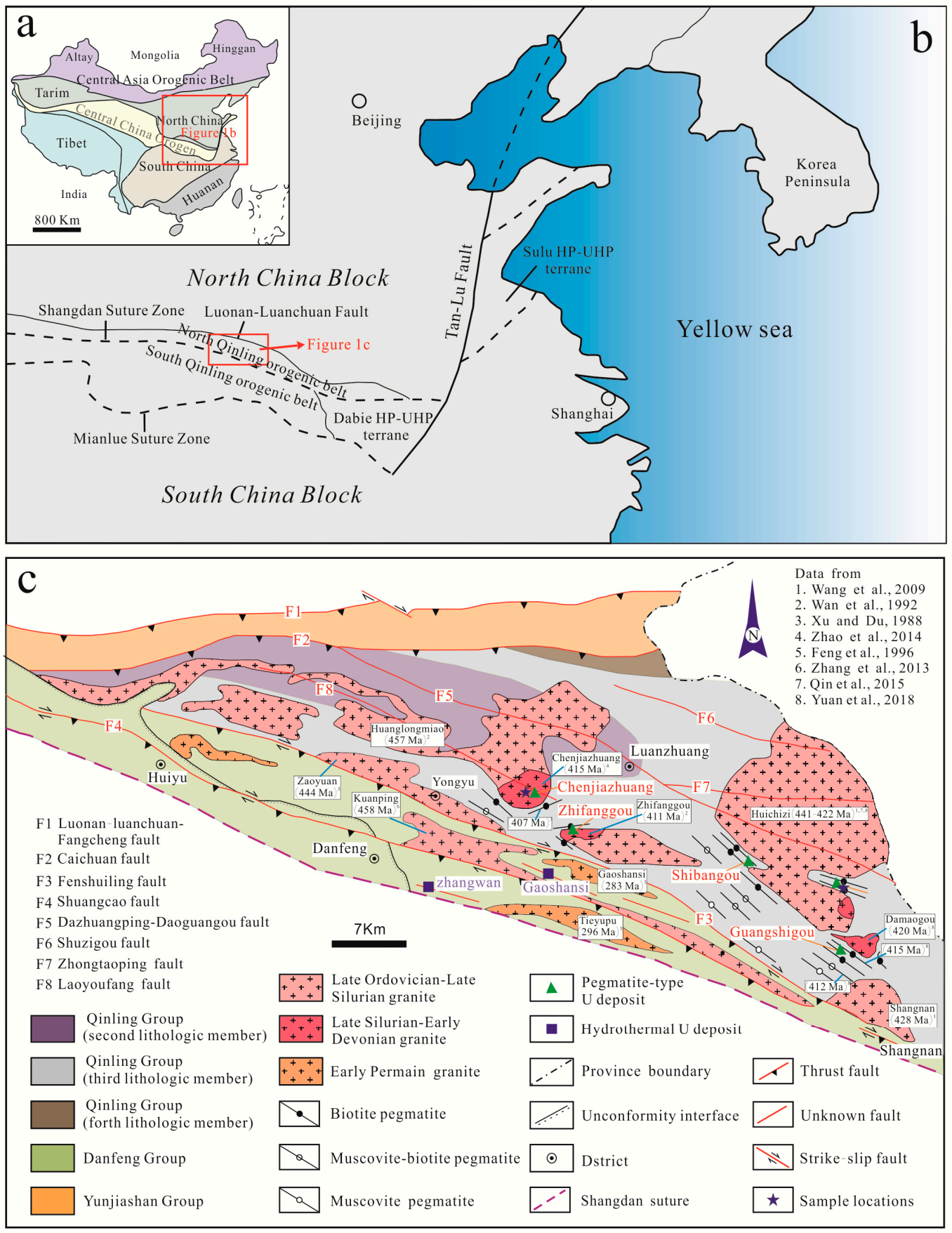

Figure 1. (a) Geotectonic units of China (modified after [33]). (b) Geological sketch map of the Qinling-Dable-Sulu orogen (modified after [34]). (c) Regional geological map of the Shangdan uraniferous province in the North Qinling orogenic belt (modified after [21]), showing the locations of pegmatite-type U deposits which are composed of the large-sized Xiaohuacha and Guangshigou deposits (>3000 t, U metal), the medium-sized Chenjiazhuang and Zhifanggou deposit (1000-3000 t, $\mathrm{U}$ metal), and the small-sized Shibangou deposit ( $<1000 \mathrm{t}$, U metal). 
Located in the central part of the NQB, the U mineralization associated with biotite pegmatites are widespread throughout the Shangdan domain, bounded on the north by the Chaichuan fault (F2 in Figure 1c) and on the south by the Fenshuiling fault (F4 in Figure 1c). The Shangdan domain represents a low-grade, large-tonnage uraniuferous province which is host to five pegmatite-type $U$ deposits, including the large-sized Xiaohuacha and Guangshigou deposits ( $>3000 t, U$ metal), the medium-sized Chenjiazhuang and Zhifanggou deposits (1000-3000 t, U metal), and the small-sized Shibangou deposit ( $<1000 \mathrm{t}, \mathrm{U}$ metal). These U deposits occur in the NNW-directed fold-and-thrust belt of the Qinling Group dominating Precambrian basement in the NQB. Although the Qinling Group rocks were proposed to be formed during the Palaeoproterozoic [30], recent detrital zircon dating suggested that they were mainly formed during the late Mesoproterozoic-early Neoproterozoic [35-37]. Regional metamorphism reached the amphibolite-greenschist facies overprinted local granulite facies [29]. Three lithologic members of the Qingling Group can be identified in the uraniferous province (Figure 1c), including a second member comprising graphite-bearing gneiss, schist, and marble; a third member comprising biotite-plagioclase gneiss and amphibolite intercalated with schist, leptynite, marble, and migmatite; and a fourth member mainly comprising marble [30]. Of these, $\mathrm{U}$ mineralization is restricted only to the contact between the biotite pegmatite and biotite-plagioclase gneiss from the third lithologic member.

The uraniferous province links the Early Paleozoic Yunjiashan Group on the north and the Neoproterozoic to Early Paleozoic Danfeng Group on the south, separated from each other by thrust faults or ductile shear zone (Figure 1c). The Yunjiashan Group consists of island arc volcanic rocks and volcanic-sedimentary rocks of the back-arc basin metamorphosed to lower greenschist-lower amphibolite facies, while the Danfeng Group is comprised of ophiolitic assemblages and subduction-related volcanic and sedimentary rocks with a metamorphic grade from greenschist to lower amphibolite facies $[27,38]$.

The uraniferous province is marked by the distribution of an Early Paleozoic NWW-directed magmatic belt (Figure 1c) which is controlled spatially and temporally by the tectono-magmatic evolution of the NQB. The granites and pegmatites were emplaced during two major episodes and evolved in different tectonic regimes. During the Late Ordovician-Late Silurian (ca. 457-422 Ma), it was intruded by volumetrically dominant calc-alkaline I-type plutons, a suite of biotite granodiorite-biotite monzogranite association [39-42]. This first stage of magmatism is coincident with or slightly later the retrograde granulite-facies metamorphism (ca. 450-440 Ma) of the Late Proterozoic eclogites and ultrahigh pressure felsic gneiss of the NQB [43], and orogen-parallel oblique westward extrusion [42], indicating a collision-induced crustal shortening, thicken, and uplift. During the Early Devonian (ca. 420-400 Ma), it was intruded by high-K calc-alkaline syenogranite and pegmatite association [22,44-46]. This second stage of magmatism synchronize with or slightly later than regional amphibolite-facies retrogression (ca. $420 \mathrm{Ma}$ ), indicating a late-stage collision and further crustal uplift [42]. Accordingly, the uraniferous province witnessed an Early Palaeozoic evolution of the NQB from deep subduction/collision to crustal uplift and exhumation [27,47].

The regional pegmatite zonation is developed with three subparallel zones concentric around the Early Devonian syenogranite: uraniferous biotite pegmatites close to the contact, biotite-muscovite pegmatites in the intermediate zone, and muscovite pegmatites in the outermost one (Figure 1c). The pegmatite swarms are commonly concordant to the strike of regional fabric observed in the adjacent metasedimentary rocks, in spite of some cross-cutting pegmatites. The undeformed nature of the uraniferous pegmatites, grouped into a NYF (niobium-yttrium-fluorine)-type family [4,48], is compatible with their late-tectonic origin. Although the Chenjiazhuang, Xiaohuacha, and Guangshigou deposits were spatially separated by several kilometers in the uraniferous province, they exhibit identical mineralization style, ore-bearing rock type (i.e. biotite pegmatite), mineral assemblages, and emplacement ages, suggest that they formed in the same metallogenic event and experienced similar U-deposition processes. 


\section{Sampling and Analytical Methods}

Representative hand specimens of uranium ores were taken from the Chenjiazhuang and Xiaohuacha biotite pegmatites in the uraniferous province, as shown in Figure 1c. Then polished thin sections were carefully examined using optical microscope with transmitted and reflected light, followed by high-contrast back-scattered electron (BSE) imaging studies for detailed mineral assemblage and microtexture observations. Besides this, uraninite separation from the Chenjiazhuang biotite pegmatite was adopt by conventional density and magnetic techniques, then handpicked under a binocular microscope.

\subsection{Isotope Dilution Thermal Ionization Mass Spectrometry (ID-TIMS)}

U-Pb ID-TIMS geochronology was performed in the ultra-clean laboratory at the Tianjin Center, China Geological Survey. Detailed analytical procedures and data acquisition were reported by Tu et al. [49]. In brief, the uraninite powder was put into acid-cleaned $1 \mathrm{~mL}$ Teflon beaker, added by $1 \mathrm{~mL} 7 \mathrm{~mol} / \mathrm{L} \mathrm{HNO}_{3}$, which was placed on a hot plate at $10{ }^{\circ} \mathrm{C}$ for $2 \mathrm{~h}$ and then rinsed with deionized water for three times. The beaker was subsequently added $1 \mathrm{~mL}$ concentrated $\mathrm{HF}$ and placed in a controlled temperature oven at $195^{\circ} \mathrm{C}$ for thoroughly sample digestion. The digested sample was divided into two parts for measuring the $\mathrm{Pb}$ isotope ratios and contents of $\mathrm{U}$ and $\mathrm{Th}$, respectively. The sample was evaporated to dryness on a hot plate at $125^{\circ} \mathrm{C}$ and then was acid diluted and loaded on outgassed Re filaments into mass spectrometer using silica gel and $\mathrm{H}_{3} \mathrm{PO}_{4}$. The $\mathrm{Pb}$ and $\mathrm{U}$ isotopic ratios were measured at currents of $2500-3000 \mathrm{~mA}$ and $2800-3300 \mathrm{~mA}$, respectively. The $\mathrm{Pb}$ isotope mass fractionation was corrected using the SRM982 $\mathrm{Pb}$ standard. Experimental data were processed by the Isoplot softwares [50], with uncertainties at the 95\% confidence level.

\subsection{Electron Probe Micro-Analysis (EPMA)}

Quantitative analyses of chemical composition of uraninite and coffinite were obtained by EPMA using a JXA-8230 (JEOL Ltd., Tokyo, Japan) instrument, equipped with a BSE detector as well as wavelength dispersive spectrometers, which was housed in the microprobe center in the Institute of Mineral Resources, Chinese Academy of Geological Sciences, Beijing. Standard operating conditions included a beam size of $5 \mu \mathrm{m}$, an electron beam current of $50 \mathrm{nA}$, and an accelerating voltage of $20 \mathrm{kV}$. The analyses were calculated using the $\mathrm{ZAF}$ correction procedure. Major $(\mathrm{U}, \mathrm{Th}, \mathrm{Pb}$, and $\mathrm{Y})$ and trace element ( $\mathrm{Si}, \mathrm{Fe}, \mathrm{Ca}, \mathrm{Al}, \mathrm{Na}, \mathrm{Mn}, \mathrm{Mg}$, and Ti) were measured. Detection limits under the analytical conditions are $<0.1 \mathrm{wt} . \%$.

\subsection{Chemical Age Dating}

Based on the decay theory of radionuclides, chemical U-Th-Pb ages can be constrained by simultaneous analysis of $\mathrm{U}, \mathrm{Th}$, and $\mathrm{Pb}$ contents determined by EPMA. The assumptions inherent to the dating method are non-radiogenic Pb being assumed as zero during crystallization and no significant $\mathrm{Pb}$ loss and reintroduction after initial crystallization [51]. It is suggested that non-radiogenic $\mathrm{Pb}$ is significantly lower than in situ Pb growth in uraninite [52,53]. Moreover, the ID-TIMS analysis confirmed that non-radiogenic $\mathrm{Pb}$ in the uraninite samples is low according to the ${ }^{204} \mathrm{~Pb}$ signal and ${ }^{207} \mathrm{~Pb} /{ }^{206} \mathrm{~Pb}$ ratios [49], and thus chemical dating can be employed to estimate the crystallization age of uraninite samples.

Single-point $\mathrm{U}-\mathrm{Pb}$ apparent ages $(\mathrm{t})$ can be calculated using the following radioactive decay Equation (1) [54]:

$$
\mathrm{Pb}=\mathrm{U} \cdot\left(0.99276 \cdot \exp \left(\left(\lambda \mathrm{U}_{238} \cdot \mathrm{t}\right)-1\right)+0.007196 \cdot \exp \left(\left(\lambda \mathrm{U}_{235} \cdot \mathrm{t}\right)-1\right)\right)+\mathrm{Th} \cdot \exp \left(\left(\lambda \mathrm{Th}_{232} \cdot \mathrm{t}\right)-1\right),
$$

where $\mathrm{Pb}, \mathrm{U}$, and Th are their contents in atomic $\%$ and $\lambda \mathrm{U}_{238}, \lambda \mathrm{U}_{235}$, and $\lambda \mathrm{Th}_{232}$ are the decay constraints of $\mathrm{U}_{238}\left(1.55125 \times 10^{-10} /\right.$ year $), \mathrm{U}_{235}\left(9.8485 \times 10^{-10} /\right.$ year $)$, and $\lambda \mathrm{Th}_{232}\left(4.9475 \times 10^{-11} /\right.$ year $)$ [55]. 
This Equation can be reorganized using the following iterative procedure:

$$
\begin{aligned}
& \mathrm{PbO}=0.848485 \cdot \mathrm{ThO}_{2} \cdot\left(\exp \left(\lambda \mathrm{Th}_{232} \cdot \mathrm{t}\right)-1\right)+\mathrm{UO}_{2} \cdot\left(0.816367 \cdot\left(\exp \left(\lambda \mathrm{U}_{238} \cdot \mathrm{t}\right)-1\right)+\right. \\
& \left.0.0059475 \cdot\left(\exp \left(\lambda \mathrm{U}_{235} \cdot \mathrm{t}\right)-1\right)\right),
\end{aligned}
$$

with $\mathrm{PbO}, \mathrm{UO}_{2}$, and $\mathrm{ThO}_{2}$ contents in uraninite. The mean error in chemical dating of uraninite is restrained to ca. $25 \mathrm{~m}$.y. by repeated measurements of the same spot and the counting statistics ([56]).

\section{Occurrence of Uraninite and Coffinite}

Disseminated uaninite and coffinite were observed in the Chenjiazhuang, Xiaohuacha, and Guangshigou biotite pegmatites when reviewed by both reflected light optical microscopy photomicrographs and BSE images (Figure 2). The uranium minerals display euhedral-subhedral cubic crystal forms for uraninite (up to $300 \mu \mathrm{m}$ in size), but an aggregate of nodular grains and veinlets for coffinite (Figure 2). Uraninite grains are intimated intergrown or textually associated with biotite aggregates (Figure 2a,b) and present as inclusions in a matrix of intimately mixed quartz and (perthitic) microcline in close association with biotite (Figure 2c). The close association of rutile (Figure 2a,c), ilmenite (Figure 2c,d), and monazite (Figure 2e) with uraninite for some grains supports a mineral paragenetic sequence. However, euhedral zircon crystals occur as xenocrysts within biotite aggregates (Figure $2 b$ ) and uraninite grains (Figure $2 \mathrm{f}$ ), suggestive zircon precipitation occurred before uraninite and biotite. Galena also occurs as either micron-sized inclusion-hosted $\mathrm{Pb}$ dispersed within uraninite (Figure 2d) or granular crystal in its vicinity (Figure 2g). In addition, anhedral quartz (Figure 2d,h), biotite, calcite (Figure 2d), and xenotime (Figure 2e) can be found within the uraninite grains. There is an embayed margin around some uraninite grains, filled by an assemblage of apatite, thorite (enclosed in apatite), calcite, and biotite (Figure 2i). It is often observed that uraninites are enveloped by radiating-fibrous pyrite (Figure $2 \mathrm{~h}$ ) and some grains heavily fractured.

Coffinite commonly shows a preferential association with uraninite and pyrite (Figure 2a,g,j-l). Pyrite grains (Figure 2g,l) and veinlets (Figure 2j,k) are often sandwiched between uraninite and coffinite rind. It can be observed that some uraninite grains are embedded in a mixed coffinite, calcite, pyrite, and galena assemblage (Figure $2 \mathrm{~g}$ ) and rimmed by coffinite aggregates (Figure 2j-1). Besides, coffinite of much lower brightness than the uraninite occurs adjacent to uraninite grain as veinlets in association with pyrite (Figure 2j,1).

The individual uraniferous pegmatites exhibit mineralogical zonation, which can be subdivided into rim, internal, and quartz core zones (see the details in [5]). Based on field and petrographic observations, there paragenetic stages are present (Figure 3): (1) the stage I (represented by the rim zone), the stage II (represented by the internal zone), and the stage III (represented by the quartz core zone). The stage I is characterized by the formation of a complex mineral association, including abundant biotite, microcline, perthite, oligoclase, quartz, and subordinate amounts of uraninite, coffinite, thorite, rutile, ilmenite, zircon, monazite, apatite, pyrite, molybdenite, galena, calcite, and alteration minerals (e.g. muscovite, albite, and hematite). The stage II is characterized by the presence of a simple mineral association, including abundant oligoclase, microcline, perthite, quartz, and minor zircon, monazite, apatite, and alteration minerals (e.g. albite). The stage III is characterized by the occurrence of massive quartz core. 

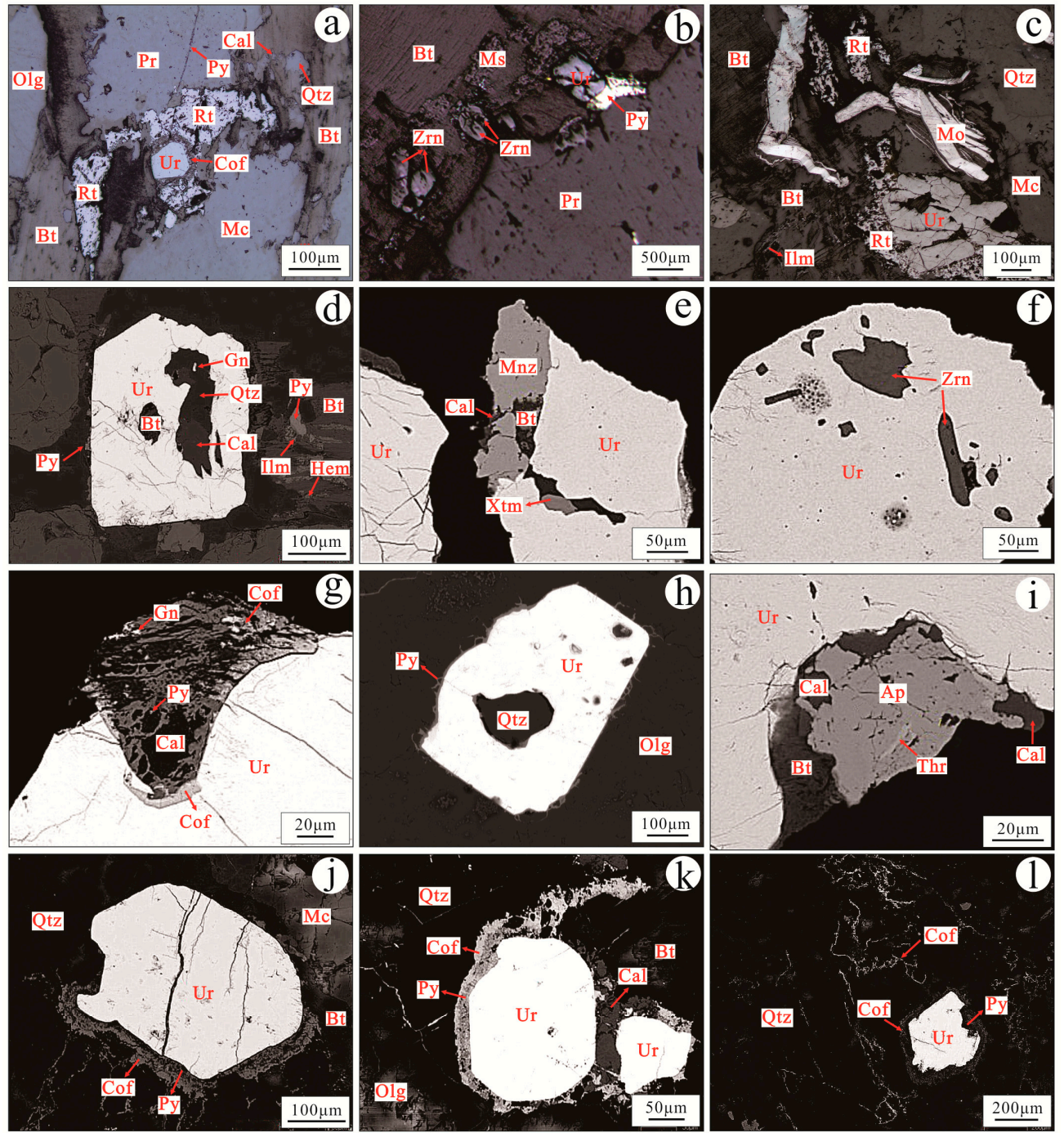

Figure 2. Representative photomicrographs (a through c) and back-scattered electron (BSE) images (d through 1) showing modes of occurrence of uraninite and coffinite from the Chenjiazhuang, Xiaohuacha, and Guangshigou biotite pegmatites. (a), (b), and (c) Microphotograph under reflected light images show the intergrowths of euhedral-subhedral uraninite with aggregated laths of biotite and uraninite occurring as inclusion within a host quartz and (perthitic) microcline in close association with biotite for the Chenjiazhuang, Guangshigou, and Xiaohuacha pegmatites, respectively; (d) galena, quartz, biotite, and calcite are present in the form of inclusions within the uraninite grain (Chenjiazhuang pegmatite, BSE image); (e) monazite occurs in close paragenesis with uraninite, and xenotime occurs in the fracture of uraninite (Xiaohuacha pegmatite, BSE image); (f) euhedral zircon clusters can be observed within the uraninite grain (Chenjiazhuang pegmatite, BSE image), suggesting zircon pre-dates uraninite; (g) an association of calcite, coffinite, galena, and pyrite is embedded in the uraninite grain (Guangshigou pegmatite, BSE image); (h) aureole of radiating-fibrous pyrite around uraninite is contained in the oligoclase host (Xiaohuacha pegmatite, BSE image); (i) uraninite grain boundary is embayed and filled with an association of apatite, thorite, biotite, and calcite (Xiaohuacha pegmatite, BSE image); (j) nodular coffinites rimming uraninite in a matrix of quartz and microcline, are in close association with pyrite (Xiaohuacha pegmatite, BSE image); (k) coffinite nucleate in the edges of uraninite and pyrite (Guangshigou pegmatite, BSE image); (1) coffinite aggregates occur as alteration rind the peripheries of uraninite and veinlets in close proximity to uraninite (Chenjiazhuang pegmatite, BSE image). Mineral abbreviations: Bt—biotite; Ms-muscovite; Qtz-quartz; Mc-microcline; Pr-perthite; Olg-oligoclase; Ur-uraninite; Cof-coffinite; Thr-thorite; Gn-galena; Mo-molybdenite; Py_pyrite; Ilm—ilmenite; Hem-hematite; Rt—rutile; Zrn—zircon; Mnz-monazite; Ap—apatite; Xtm-xenotime; Cal—calcite. 


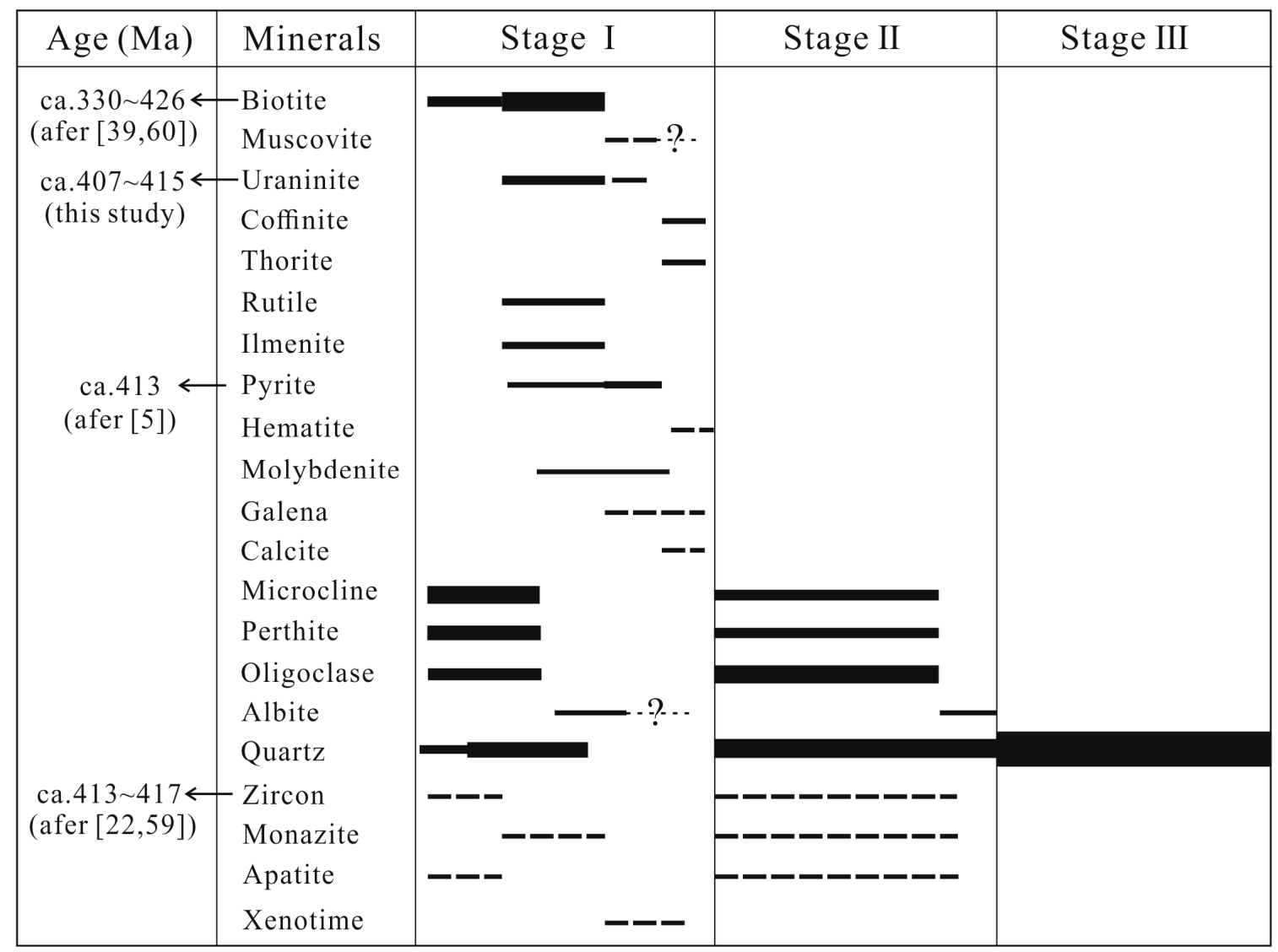

Figure 3. Paragenetic sequence diagram for mineral assemblages in the Chenjiazhuang, Xiaohuacha, and Guangshigou biotite pegmatites. The widths of lines indicate relative abundances of minerals. Dashed lines show minor and trace phases.

\section{Results}

\subsection{Isotope Dilution Thermal Ionization Mass Spectrometry (ID-TIMS) Ages}

Three fractions of uraninite from the Chenjiazhuang biotite pegmatite (sample CJZ-1) were analyzed by ID-TIMS and the U-Pb data were listed in Table 1 . They have significant $\mathrm{U}$ and $\mathrm{Pb}$ concentrations in the range of 78.76-88.09 wt.\% and 4.73-5.27 wt.\%, respectively. All three fractions plot in a concordant position in a concordia diagram of ${ }^{206} \mathrm{~Pb}^{238} \mathrm{U}$ and ${ }^{207} \mathrm{~Pb}^{235} \mathrm{U}$ (Figure 4) and allow calculate a concordia age of $406.8 \pm 0.5 \mathrm{Ma}(\mathrm{n}=3, \mathrm{MSWD}=0.68)$, with a weighted mean age of $406.5 \pm 1.2 \mathrm{Ma}(\mathrm{n}=3, \mathrm{MSWD}=0.87)$.

Table 1. Isotope dilution thermal ionization mass spectrometry (ID-TIMS) uraninite U-Pb isotopic data for the Chenjiazhuang biotite pegmatite.

\begin{tabular}{|c|c|c|c|c|c|c|c|c|c|c|c|c|c|c|c|}
\hline \multirow[b]{2}{*}{ Fraction } & \multirow[b]{2}{*}{$\begin{array}{l}\text { Weight } \\
\text { (mg) }\end{array}$} & \multicolumn{2}{|c|}{ Concentrations } & \multicolumn{8}{|c|}{ Isotopic Ratios } & \multicolumn{4}{|c|}{ Apparent Age (Ma) } \\
\hline & & $\underset{\text { (wt.\%) }}{\mathrm{U}}$ & $\begin{array}{c}\mathrm{Pb} \\
\text { (wt.\%) }\end{array}$ & $\begin{array}{l}{ }^{206} \mathrm{~Pb} / \\
{ }^{204} \mathrm{~Pb}\end{array}$ & $\begin{array}{c}\text { Err } \\
\text { (wt.\%) }\end{array}$ & $\begin{array}{l}{ }^{206} \mathrm{~Pb} / \\
{ }^{238} \mathrm{U}\end{array}$ & $\begin{array}{c}\text { Err } \\
\text { (wt.\%) }\end{array}$ & $\begin{array}{c}{ }^{207} \mathrm{~Pb} / \\
{ }^{235} \mathrm{U}\end{array}$ & $\begin{array}{c}\text { Err } \\
\text { (wt.\%) }\end{array}$ & $\begin{array}{l}{ }^{207} \mathrm{~Pb} / \\
{ }^{206} \mathrm{~Pb}\end{array}$ & $\begin{array}{c}\text { Err } \\
\text { (wt.\%) }\end{array}$ & $\begin{array}{l}{ }^{{ }^{206}} \mathrm{~Pb} / \\
{ }^{238} \mathrm{U}\end{array}$ & $\pm 1 \sigma$ & $\begin{array}{c}{ }^{207} \mathrm{~Pb} / \\
{ }^{235} \mathrm{U}\end{array}$ & $\pm 1 \sigma$ \\
\hline CIZ-1-1 & 70 & 78.76 & 4.73 & 8073 & 0.04 & 0.06510 & 0.17 & 0.4933 & 0.17 & 0.0549 & 0.05 & 406.6 & 0.7 & 407.1 & 0.7 \\
\hline CJZ-1-2 & 70 & 82.26 & 4.89 & 46719 & 0.09 & 0.06478 & 0.42 & 0.4905 & 0.42 & 0.0549 & 0.02 & 404.6 & 1.7 & 405.2 & 1.7 \\
\hline CJZ-1-3 & 100 & 88.09 & 5.27 & 138375 & 0.17 & 0.06530 & 0.44 & 0.4944 & 0.44 & 0.0549 & 0.02 & 407.8 & 1.8 & 407.9 & 1.8 \\
\hline
\end{tabular}



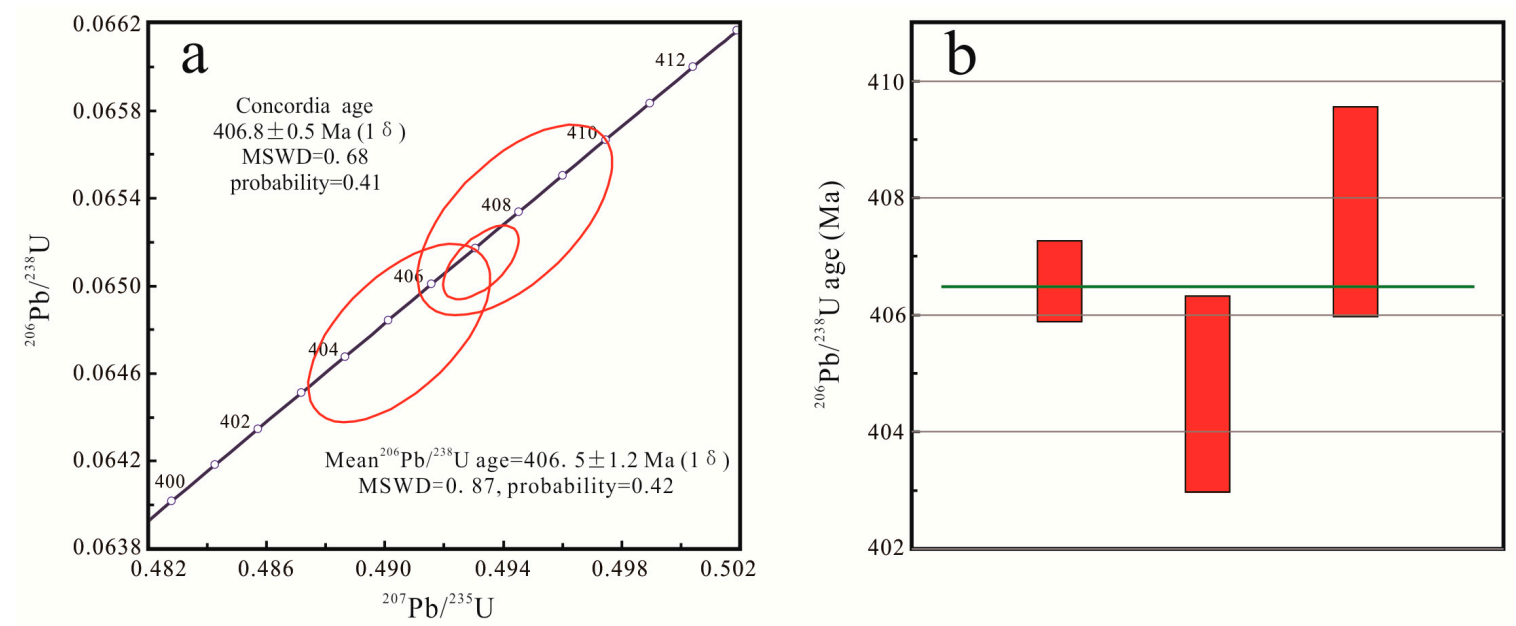

Figure 4. U-Pb concordia diagram (a) and weighted diagram (b) for isotope dilution thermal ionization mass spectrometry (ID-TIMS) uraninite analyses from the Chenjiazhuang biotite pegmatite.

\subsection{Chemical Compositions of Uraninite and Coffinite}

The compositional data of uraninite and coffinite from the Chenjiazhuang and Xiaohuacha biotite pegmatites were presented in Table 2 and a statistical treatment of the chemical composition of uraninite (cf. [11,57]) was performed in Table 3. There is no discernible variation in BSE brightness of uraninite (Figure 2), which can be used to link compositional changes to different texture types (e.g., large grain vs. small grain, euhedral crystal vs. subhedral crystal, and uraninite associated with different mineral association), while they are distinguished from coffinite of different textural type by BSE brightness and contrast in terms of chemical compositions.

The analytical totals of uraninite range from 91.92 to $99.11 \mathrm{wt} . \%$. Partial oxidation of $\mathrm{U}^{4+}$ to $\mathrm{U}^{6+}$ by self-oxidation and valence compensation by cationic substitutions are important in the interpretation of the results of some low total values. The presence of $\mathrm{U}^{6+}$ can also be supported by estimated total positive charges $(<4.0)$ based on 1 cation per formula unit [53]. According to the major elements $U$, Th, and $Y$, uraninite can be classified into two distinct chemical groups (Figure 5): uraninite-A with high $\mathrm{ThO}_{2}$ (2.07-8.37 wt.\%, average $3.19 \pm 0.85 \mathrm{wt.} \%)$, but low $\mathrm{UO}_{2}(78.82-90.92 \mathrm{wt} . \%$, average $85.87 \pm 1.80 \mathrm{wt} . \%)$ and variable $\mathrm{Y}_{2} \mathrm{O}_{3}$ contents $(0.29-1.49 \mathrm{wt} . \%$, average $0.81 \pm 0.24 \mathrm{wt} . \%)$; uraninite-B with restricted but low $\mathrm{ThO}_{2}(0.18-0.41 \mathrm{wt} . \%$, average $0.28 \pm 0.08 \mathrm{wt} . \%)$, and high $\mathrm{UO}_{2}(87.64-90.65 \mathrm{wt} . \%$, average $88.88 \pm 0.96 \mathrm{wt} . \%))$ and $\mathrm{Y}_{2} \mathrm{O}_{3}$ contents $(0.79-1.4 \mathrm{wt} . \%$, average $1.05 \pm 0.19 \mathrm{wt} . \%)$. The Xiaohuacha uraninite-A typical has elevated $\mathrm{ThO}_{2}(4.15-8.37 \mathrm{wt} . \%$, average $5.04 \pm 1.15 \mathrm{wt} . \%)$ but lower $\mathrm{UO}_{2}$ (78.82-87.77 wt.\%, average $83.43 \pm 2.60$ wt.\%) compared to the Chenjiazhuang and Guangshigou uraninite- $\mathrm{A}\left(\mathrm{ThO}_{2}=2.07-4.41 \mathrm{wt} . \%\right.$, average $2.95 \pm 0.39 \mathrm{wt} . \% ; \mathrm{UO}_{2}=82.64-90.62 \mathrm{wt} . \%$, average $86.18 \pm 1.41 \mathrm{wt} . \%$, Figure 5a). The Chenjiazhuang, Xiaohuacha, and Guangshigou uraninite-A and uraninite-B have consistent mean $\mathrm{PbO}$ contents $(4.79 \pm 0.26$ wt. $\%, 4.65 \pm 0.78$ wt. $\%, 4.85 \pm 0.23$ wt. $\%$, and $4.5 \pm 0.19 \mathrm{wt} . \%$, Figure $5 \mathrm{c}$ ), although a few of abnormally high-Pb analyses more likely reflect contamination from galena micro-inclusions. Note that the uraninite-A were widely distributed in the Chenjiazhuang, Xiaohuacha, and Guangshigou biotite pegmatites, whereas the uraninite-B were only found in the Guangshigou biotite pegmatites. 
Table 2. Chemical data of uraninite and coffinite and calculated chemical ages of uraninite from the Chenjiazhuang and Xiaohuacha biotite pegmatites in the uraniferous province.

\begin{tabular}{|c|c|c|c|c|c|c|c|c|c|c|c|c|c|c|c|}
\hline NO. & $\mathrm{SiO}_{2}$ & $\mathrm{CaO}$ & $\mathrm{Al}_{2} \mathrm{O}_{3}$ & $\mathrm{FeO}$ & $\mathrm{MgO}$ & $\mathrm{Na}_{2} \mathrm{O}$ & $\mathrm{MnO}$ & $\mathrm{TiO}_{2}$ & $\mathrm{ThO}_{2}$ & $\mathrm{UO}_{2}$ & $\mathrm{PbO}$ & $\mathrm{Y}_{2} \mathrm{O}_{3}$ & $\mathrm{Ce}_{2} \mathrm{O}_{3}$ & Total & Chemical Age (Ma) \\
\hline \multicolumn{16}{|c|}{ Chenjiazhuang biotite pegmatite (uraninite) } \\
\hline 1 & 0.09 & 0.22 & 0.00 & 0.00 & 0.02 & 0.09 & 0.00 & 0.00 & 2.54 & 86.73 & 4.68 & 0.99 & 0.13 & 95.53 & 388 \\
\hline 2 & 0.12 & 0.14 & 0.00 & 0.05 & 0.00 & 0.08 & 0.00 & 0.00 & 2.55 & 86.25 & 4.56 & 0.97 & 0.11 & 94.91 & 380 \\
\hline 3 & 0.83 & 0.47 & 0.00 & 0.00 & 0.01 & 0.07 & 0.00 & 0.00 & 2.43 & 83.60 & 4.63 & 0.98 & 0.11 & 93.20 & 398 \\
\hline 4 & 0.06 & 0.03 & 0.02 & 0.02 & 0.00 & 0.17 & 0.03 & 0.00 & 2.63 & 88.43 & 4.54 & 0.51 & 0.14 & 96.65 & 370 \\
\hline 5 & 0.10 & 0.24 & 0.00 & 0.00 & 0.01 & 0.09 & 0.02 & 0.02 & 2.74 & 86.62 & 4.33 & 1.20 & 0.23 & 95.88 & 360 \\
\hline 6 & 0.00 & 0.23 & 0.00 & 0.06 & 0.03 & 0.09 & 0.00 & 0.00 & 2.73 & 85.72 & 4.85 & 1.07 & 0.20 & 95.22 & 406 \\
\hline 7 & 0.00 & 0.19 & 0.00 & 0.01 & 0.03 & 0.18 & 0.02 & 0.00 & 2.33 & 87.43 & 4.77 & 0.74 & 0.18 & 95.97 & 392 \\
\hline 8 & 0.33 & 0.28 & 0.00 & 0.01 & 0.02 & 0.08 & 0.00 & 0.00 & 2.44 & 83.74 & 5.43 & 0.89 & 0.23 & 93.51 & 463 \\
\hline 9 & 0.27 & 0.09 & 0.00 & 0.04 & 0.01 & 0.12 & 0.04 & 0.00 & 2.42 & 84.42 & 5.42 & 0.87 & 0.13 & 93.95 & 458 \\
\hline 10 & 0.33 & 0.26 & 0.00 & 0.07 & 0.01 & 0.08 & 0.02 & 0.04 & 2.32 & 83.62 & 5.65 & 1.03 & 0.30 & 93.92 & 482 \\
\hline 11 & 0.09 & 0.07 & 0.00 & 0.03 & 0.02 & 0.08 & 0.02 & 0.00 & 3.43 & 83.62 & 4.72 & 1.01 & 0.17 & 93.35 & 404 \\
\hline 12 & 0.08 & 0.17 & 0.12 & 0.05 & 0.01 & 0.06 & 0.00 & 0.00 & 2.65 & 85.32 & 4.72 & 0.88 & 0.10 & 94.38 & 397 \\
\hline 13 & 0.51 & 0.37 & 0.01 & 0.27 & 0.00 & 0.04 & 0.02 & 0.00 & 2.64 & 83.64 & 4.82 & 0.80 & 0.26 & 93.46 & 413 \\
\hline 14 & 0.59 & 0.53 & 0.00 & 0.03 & 0.00 & 0.10 & 0.00 & 0.00 & 2.85 & 82.88 & 4.74 & 0.90 & 0.26 & 93.02 & 410 \\
\hline 15 & 0.12 & 0.14 & 0.08 & 0.08 & 0.03 & 0.05 & 0.02 & 0.00 & 2.85 & 84.65 & 4.84 & 0.92 & 0.16 & 94.26 & 410 \\
\hline 16 & 0.73 & 0.48 & 0.00 & 0.06 & 0.01 & 0.10 & 0.00 & 0.00 & 2.75 & 83.63 & 4.89 & 1.06 & 0.26 & 93.04 & 419 \\
\hline 17 & 0.54 & 0.48 & 0.00 & 0.07 & 0.00 & 0.04 & 0.00 & 0.00 & 2.72 & 87.25 & 4.84 & 1.02 & 0.17 & 96.14 & 398 \\
\hline 18 & 0.90 & 0.76 & 0.00 & 0.03 & 0.02 & 0.04 & 0.00 & 0.00 & 2.86 & 83.32 & 4.82 & 1.19 & 0.35 & 93.46 & 414 \\
\hline 19 & 0.00 & 0.13 & 0.00 & 0.00 & 0.02 & 0.13 & 0.00 & 0.00 & 2.98 & 86.72 & 4.55 & 0.67 & 0.13 & 95.44 & 377 \\
\hline 20 & 0.22 & 0.19 & 0.00 & 0.21 & 0.02 & 0.07 & 0.00 & 0.00 & 3.24 & 84.42 & 4.72 & 0.80 & 0.14 & 94.03 & 400 \\
\hline 21 & 0.28 & 0.19 & 0.00 & 0.09 & 0.04 & 0.07 & 0.00 & 0.04 & 2.78 & 84.53 & 4.85 & 0.62 & 0.20 & 93.69 & 411 \\
\hline 22 & 0.00 & 0.08 & 0.00 & 0.05 & 0.02 & 0.10 & 0.05 & 0.00 & 2.82 & 87.02 & 4.46 & 0.70 & 0.16 & 95.46 & 369 \\
\hline 23 & 0.01 & 0.09 & 0.00 & 0.00 & 0.02 & 0.09 & 0.03 & 0.00 & 2.54 & 86.72 & 4.63 & 0.97 & 0.10 & 95.20 & 384 \\
\hline 24 & 0.12 & 0.07 & 0.00 & 0.06 & 0.02 & 0.09 & 0.00 & 0.00 & 3.02 & 85.70 & 4.66 & 0.78 & 0.20 & 94.72 & 390 \\
\hline 25 & 0.05 & 0.28 & 0.00 & 0.01 & 0.02 & 0.14 & 0.02 & 0.00 & 2.72 & 87.62 & 4.73 & 0.73 & 0.19 & 96.51 & 388 \\
\hline 26 & 0.03 & 0.20 & 0.00 & 0.00 & 0.02 & 0.09 & 0.00 & 0.00 & 2.82 & 88.01 & 4.62 & 0.60 & 0.10 & 96.49 & 377 \\
\hline 27 & 0.14 & 0.26 & 0.00 & 0.11 & 0.00 & 0.08 & 0.00 & 0.01 & 2.54 & 85.65 & 4.54 & 0.96 & 0.10 & 94.39 & 381 \\
\hline 28 & 0.07 & 0.17 & 0.00 & 0.63 & 0.03 & 0.13 & 0.03 & 0.00 & 2.75 & 84.65 & 4.78 & 0.85 & 0.22 & 94.31 & 405 \\
\hline 29 & 0.01 & 0.22 & 0.00 & 0.03 & 0.02 & 0.11 & 0.00 & 0.04 & 2.72 & 86.53 & 4.86 & 1.04 & 0.24 & 95.82 & 403 \\
\hline 30 & 0.00 & 0.27 & 0.00 & 0.03 & 0.02 & 0.11 & 0.00 & 0.00 & 2.82 & 87.66 & 4.64 & 0.89 & 0.13 & 96.57 & 381 \\
\hline 31 & 0.05 & 0.41 & 0.00 & 0.07 & 0.00 & 0.08 & 0.03 & 0.00 & 2.62 & 85.62 & 4.80 & 0.96 & 0.22 & 94.86 & 402 \\
\hline 32 & 0.02 & 0.37 & 0.00 & 0.00 & 0.02 & 0.13 & 0.00 & 0.00 & 2.58 & 86.42 & 4.77 & 0.85 & 0.24 & 95.40 & 396 \\
\hline 33 & 0.05 & 0.28 & 0.01 & 0.00 & 0.00 & 0.14 & 0.00 & 0.00 & 2.82 & 85.82 & 4.55 & 0.94 & 0.25 & 94.86 & 381 \\
\hline 34 & 0.05 & 0.27 & 0.00 & 0.04 & 0.02 & 0.16 & 0.03 & 0.00 & 2.72 & 85.72 & 5.02 & 1.14 & 0.21 & 95.38 & 419 \\
\hline 35 & 0.01 & 0.33 & 0.00 & 0.05 & 0.02 & 0.09 & 0.00 & 0.00 & 2.56 & 85.54 & 4.65 & 1.16 & 0.15 & 94.56 & 391 \\
\hline 36 & 0.05 & 0.29 & 0.08 & 0.05 & 0.03 & 0.14 & 0.01 & 0.00 & 2.63 & 85.75 & 5.12 & 0.96 & 0.05 & 95.16 & 427 \\
\hline
\end{tabular}


Table 2. Cont.

\begin{tabular}{|c|c|c|c|c|c|c|c|c|c|c|c|c|c|c|c|}
\hline NO. & $\mathrm{SiO}_{2}$ & $\mathrm{CaO}$ & $\mathrm{Al}_{2} \mathrm{O}_{3}$ & $\mathrm{FeO}$ & $\mathrm{MgO}$ & $\mathrm{Na}_{2} \mathrm{O}$ & $\mathrm{MnO}$ & $\mathrm{TiO}_{2}$ & $\mathrm{ThO}_{2}$ & $\mathrm{UO}_{2}$ & $\mathrm{PbO}$ & $\mathrm{Y}_{2} \mathrm{O}_{3}$ & $\mathrm{Ce}_{2} \mathrm{O}_{3}$ & Total & Chemical Age (Ma) \\
\hline \multicolumn{16}{|c|}{ Chenjiazhuang biotite pegmatite (uraninite) } \\
\hline 37 & 0.58 & 0.37 & 0.05 & 0.03 & 0.02 & 0.05 & 0.00 & 0.00 & 2.41 & 84.96 & 4.72 & 0.97 & 0.15 & 94.31 & 399 \\
\hline 38 & 0.03 & 0.25 & 0.00 & 0.37 & 0.03 & 0.13 & 0.00 & 0.00 & 2.79 & 85.54 & 5.06 & 0.96 & 0.18 & 95.34 & 423 \\
\hline 39 & 0.55 & 0.64 & 0.00 & 0.09 & 0.02 & 0.09 & 0.00 & 0.00 & 3.54 & 82.67 & 4.65 & 0.75 & 0.15 & 93.15 & 402 \\
\hline 40 & 0.75 & 0.64 & 0.00 & 0.06 & 0.02 & 0.08 & 0.03 & 0.02 & 3.43 & 82.64 & 4.73 & 0.86 & 0.10 & 93.36 & 409 \\
\hline 41 & 0.80 & 0.53 & 0.00 & 0.03 & 0.00 & 0.10 & 0.05 & 0.00 & 2.82 & 85.94 & 5.04 & 0.87 & 0.16 & 96.34 & 420 \\
\hline 42 & 0.67 & 0.43 & 0.00 & 0.00 & 0.01 & 0.15 & 0.00 & 0.00 & 2.99 & 84.62 & 4.83 & 0.86 & 0.19 & 94.75 & 409 \\
\hline Average & 0.24 & 0.29 & 0.01 & 0.07 & 0.02 & 0.10 & 0.01 & 0.00 & 2.75 & 85.41 & 4.79 & 0.90 & 0.18 & 94.76 & \\
\hline $\pm 1 \sigma$ & 0.28 & 0.17 & 0.02 & 0.11 & 0.01 & 0.03 & 0.01 & 0.01 & 0.28 & 1.53 & 0.26 & 0.16 & 0.06 & 1.09 & \\
\hline \multicolumn{16}{|c|}{ Chenjiazhuang biotite pegmatite (coffinite) } \\
\hline 1 & 7.74 & 3.06 & 0.29 & 1.12 & 0.18 & 0.15 & 0.00 & 0.05 & 1.46 & 75.45 & 0.07 & 1.16 & 0.36 & 91.08 & \\
\hline 2 & 5.34 & 1.32 & 0.17 & 0.27 & 0.13 & 0.00 & 0.00 & 0.18 & 3.34 & 76.66 & 2.15 & 0.68 & 0.00 & 90.23 & \\
\hline Average & 6.54 & 2.19 & 0.46 & 0.70 & 0.16 & 0.08 & 0.00 & 0.12 & 2.40 & 76.06 & 1.11 & 0.92 & 0.18 & 90.66 & \\
\hline $\pm 1 \sigma$ & 1.70 & 1.23 & 0.08 & 0.60 & 0.04 & 0.11 & 0.00 & 0.09 & 1.33 & 0.86 & 1.47 & 0.34 & 0.25 & 0.60 & \\
\hline \multicolumn{16}{|c|}{ Xiaohuacha biotite pegmatite (uraninite) } \\
\hline 1 & 0.36 & 0.05 & 0.00 & 0.64 & 0.06 & 0.00 & 0.00 & 0.00 & 8.37 & 78.82 & 4.56 & 1.08 & 0.19 & 94.13 & 406 \\
\hline 2 & 0.09 & 0.21 & 0.00 & 0.07 & 0.05 & 0.14 & 0.09 & 0.00 & 5.14 & 83.30 & 3.36 & 0.99 & 0.08 & 93.51 & 290 \\
\hline 3 & 0.06 & 0.20 & 0.00 & 0.04 & 0.00 & 0.07 & 0.01 & 0.00 & 5.18 & 84.25 & 3.35 & 1.02 & 0.06 & 94.25 & 286 \\
\hline 4 & 0.11 & 0.25 & 0.00 & 0.06 & 0.00 & 0.11 & 0.04 & 0.00 & 5.01 & 84.95 & 4.77 & 0.88 & 0.18 & 96.34 & 399 \\
\hline 5 & 0.00 & 0.12 & 0.03 & 0.07 & 0.00 & 0.06 & 0.00 & 0.00 & 4.95 & 80.78 & 5.69 & 1.05 & 0.09 & 92.84 & 496 \\
\hline 6 & 0.07 & 0.21 & 0.23 & 0.02 & 0.02 & 0.05 & 0.00 & 0.00 & 5.13 & 81.88 & 5.07 & 1.02 & 0.38 & 94.07 & 438 \\
\hline 7 & 0.07 & 0.21 & 0.16 & 0.06 & 0.00 & 0.03 & 0.00 & 0.00 & 6.68 & 80.57 & 4.85 & 1.09 & 0.13 & 93.85 & 424 \\
\hline 8 & 0.05 & 0.03 & 0.00 & 0.00 & 0.00 & 0.08 & 0.00 & 0.00 & 4.30 & 85.00 & 3.57 & 0.87 & 0.18 & 94.07 & 302 \\
\hline 9 & 0.05 & 0.14 & 0.01 & 0.02 & 0.03 & 0.01 & 0.00 & 0.00 & 3.69 & 87.77 & 4.76 & 0.63 & 0.15 & 97.27 & 389 \\
\hline 10 & 0.07 & 0.19 & 0.00 & 0.01 & 0.02 & 0.00 & 0.00 & 0.00 & 3.51 & 86.14 & 4.84 & 0.81 & 0.15 & 95.75 & 402 \\
\hline 11 & 0.05 & 0.08 & 0.00 & 0.00 & 0.00 & 0.24 & 0.10 & 0.00 & 4.20 & 86.76 & 4.91 & 0.72 & 0.16 & 97.23 & 404 \\
\hline 12 & 0.03 & 0.01 & 0.02 & 0.01 & 0.03 & 0.12 & 0.02 & 0.00 & 4.15 & 86.47 & 5.10 & 0.70 & 0.19 & 96.86 & 421 \\
\hline 13 & 0.00 & 0.06 & 0.00 & 0.12 & 0.01 & 0.09 & 0.00 & 0.06 & 5.39 & 84.38 & 4.79 & 1.45 & 0.17 & 96.50 & 403 \\
\hline 14 & 0.07 & 0.18 & 0.00 & 0.00 & 0.04 & 0.02 & 0.00 & 0.00 & 5.57 & 80.80 & 4.03 & 1.04 & 0.18 & 91.92 & 355 \\
\hline 15 & 0.00 & 0.17 & 0.02 & 0.11 & 0.03 & 0.05 & 0.00 & 0.13 & 4.62 & 80.70 & 6.39 & 0.72 & 0.20 & 93.14 & 555 \\
\hline 16 & 0.06 & 0.11 & 0.00 & 0.04 & 0.03 & 0.13 & 0.05 & 0.06 & 5.38 & 83.78 & 4.35 & 0.99 & 0.10 & 95.07 & 370 \\
\hline 17 & 0.20 & 0.23 & 0.00 & 0.04 & 0.02 & 0.43 & 0.00 & 0.00 & 4.46 & 81.92 & 4.66 & 0.68 & 0.22 & 92.87 & 406 \\
\hline Average & 0.08 & 0.14 & 0.01 & 0.08 & 0.02 & 0.10 & 0.01 & 0.01 & 5.04 & 83.43 & 4.65 & 0.93 & 0.17 & 94.69 & \\
\hline $\pm 1 \sigma$ & 0.09 & 0.08 & 0.06 & 0.15 & 0.02 & 0.11 & 0.03 & 0.03 & 1.15 & 2.60 & 0.78 & 0.21 & 0.07 & 1.68 & \\
\hline
\end{tabular}


Table 3. Correlation diagram for the analyzed uraninite from the Chenjiazhuang and Xiaohuacha biotite pegmatites in the uraniferous province.

\begin{tabular}{|c|c|c|c|c|c|c|c|c|c|c|c|c|c|}
\hline \multicolumn{14}{|c|}{ Chenjiazhuang Biotite Pegmatite } \\
\hline & $\mathrm{UO}_{2}$ & $\mathrm{ThO}_{2}$ & $\mathrm{PbO}$ & $\mathrm{SiO}_{2}$ & $\mathrm{CaO}$ & $\mathrm{FeO}$ & $\mathrm{Al}_{2} \mathrm{O}_{3}$ & $\mathrm{MgO}$ & $\mathrm{Na}_{2} \mathrm{O}$ & $\mathrm{MnO}$ & $\mathrm{TiO}_{2}$ & $\mathrm{Y}_{2} \mathrm{O}_{3}$ & $\mathrm{Ce}_{2} \mathrm{O}_{3}$ \\
\hline $\mathrm{UO}_{2}$ & 1.00 & & & & & & & & & & & & \\
\hline $\mathrm{ThO}_{2}$ & -0.20 & 1.00 & & & & & & & & & & & \\
\hline $\mathrm{PbO}$ & -0.36 & -0.13 & 1.00 & & & & & & & & & & \\
\hline $\mathrm{SiO}_{2}$ & -0.69 & 0.12 & 0.25 & 1.00 & & & & & & & & & \\
\hline $\mathrm{CaO}$ & -0.39 & 0.06 & 0.28 & 0.53 & 1.00 & & & & & & & & \\
\hline $\mathrm{FeO}$ & -0.41 & 0.15 & 0.33 & 0.18 & 0.02 & 1.00 & & & & & & & \\
\hline $\mathrm{Al}_{2} \mathrm{O}_{3}$ & 0.02 & -0.12 & -0.03 & 0.02 & -0.09 & 0.06 & 1.00 & & & & & & \\
\hline $\mathrm{MgO}$ & 0.03 & 0.16 & 0.22 & -0.40 & -0.26 & 0.12 & -0.08 & 1.00 & & & & & \\
\hline $\mathrm{Na}_{2} \mathrm{O}$ & 0.44 & 0.02 & 0.01 & -0.49 & -0.15 & -0.36 & -0.11 & 0.14 & 1.00 & & & & \\
\hline $\mathrm{MnO}$ & 0.10 & -0.10 & 0.11 & -0.09 & -0.22 & 0.07 & 0.06 & -0.05 & 0.22 & 1.00 & & & \\
\hline $\mathrm{TiO}_{2}$ & -0.13 & -0.07 & 0.06 & 0.13 & 0.01 & 0.17 & -0.18 & 0.00 & -0.18 & 0.04 & 1.00 & & \\
\hline $\mathrm{Y}_{2} \mathrm{O}_{3}$ & -0.18 & -0.26 & 0.21 & 0.12 & 0.27 & -0.01 & -0.10 & -0.12 & -0.25 & -0.14 & 0.15 & 1.00 & \\
\hline $\mathrm{Ce}_{2} \mathrm{O}_{3}$ & -0.25 & 0.09 & 0.40 & 0.15 & 0.28 & 0.08 & -0.15 & -0.08 & 0.05 & -0.03 & 0.12 & 0.22 & 1.00 \\
\hline \multicolumn{14}{|c|}{ Xiaohuacha Biotite Pegmatite } \\
\hline & $\mathrm{UO}_{2}$ & $\mathrm{ThO}_{2}$ & $\mathrm{PbO}$ & $\mathrm{SiO}_{2}$ & $\mathrm{CaO}$ & $\mathrm{FeO}$ & $\mathrm{Al}_{2} \mathrm{O}_{3}$ & $\mathrm{MgO}$ & $\mathrm{Na}_{2} \mathrm{O}$ & $\mathrm{MnO}$ & $\mathrm{TiO}_{2}$ & $\mathrm{Y}_{2} \mathrm{O}_{3}$ & $\mathrm{Ce}_{2} \mathrm{O}_{3}$ \\
\hline $\mathrm{UO}_{2}$ & 1.00 & & & & & & & & & & & & \\
\hline $\mathrm{ThO}_{2}$ & -0.71 & 1.00 & & & & & & & & & & & \\
\hline $\mathrm{PbO}$ & -0.07 & -0.33 & 1.00 & & & & & & & & & & \\
\hline $\mathrm{SiO}_{2}$ & -0.28 & 0.32 & -0.47 & 1.00 & & & & & & & & & \\
\hline $\mathrm{CaO}$ & -0.25 & 0.12 & -0.11 & 0.58 & 1.00 & & & & & & & & \\
\hline $\mathrm{FeO}$ & -0.58 & 0.51 & 0.10 & 0.06 & 0.12 & 1.00 & & & & & & & \\
\hline $\mathrm{Al}_{2} \mathrm{O}_{3}$ & -0.29 & -0.09 & 0.71 & -0.34 & 0.06 & 0.10 & 1.00 & & & & & & \\
\hline $\mathrm{MgO}$ & -0.22 & 0.17 & -0.19 & 0.26 & -0.14 & 0.15 & -0.09 & 1.00 & & & & & \\
\hline $\mathrm{Na}_{2} \mathrm{O}$ & 0.29 & -0.14 & -0.11 & -0.05 & 0.05 & -0.06 & -0.29 & -0.21 & 1.00 & & & & \\
\hline $\mathrm{MnO}$ & 0.40 & -0.07 & -0.20 & 0.07 & 0.01 & -0.13 & -0.34 & -0.03 & 0.67 & 1.00 & & & \\
\hline $\mathrm{TiO}_{2}$ & -0.15 & 0.20 & 0.19 & -0.47 & -0.24 & 0.42 & -0.02 & 0.17 & 0.12 & -0.02 & 1.00 & & \\
\hline $\mathrm{Y}_{2} \mathrm{O}_{3}$ & -0.60 & 0.85 & -0.12 & 0.09 & 0.00 & 0.47 & 0.05 & -0.11 & -0.29 & -0.22 & 0.09 & 1.00 & \\
\hline $\mathrm{Ce}_{2} \mathrm{O}_{3}$ & -0.15 & -0.12 & 0.34 & 0.15 & -0.05 & -0.09 & 0.17 & 0.23 & -0.04 & -0.36 & 0.06 & -0.26 & 1.00 \\
\hline
\end{tabular}

The Spearman Rank correlation coefficient is shown in the correlation diagram.

Other elemental impurities can be integrated into the uraninite lattice though primary incorporation at the time of initial crystallization and elemental substitutions during the fluid-driven post-crystallization alterations $[11,19]$. The sums of $\mathrm{SiO}_{2}, \mathrm{CaO}$, and $\mathrm{FeO}$ of uraninte- $\mathrm{B}$ are restricted and lower than $0.5 \mathrm{wt} . \%$, while those of the uraninite-A are relatively scattered $(0.01-1.69 \mathrm{wt} . \%$, Figure $5 \mathrm{~d})$. Some analyses for the uraninite-A with the sums $>0.5 \mathrm{wt} . \%$ seem to imply a post-magmatic interaction with fluids [11]. Several abnormally high-Fe analyses may be also caused by Fe excitation of Fe-bearing phase hosting uraninite grains during analysis. The uraninite can also accommodate $\mathrm{Na}_{2} \mathrm{O}$ up to $0.43 \mathrm{wt} . \%$ for the uraninite-A and $0.17 \mathrm{wt} . \%$ for the uraninite- $\mathrm{B}$ (Figure 5e). The contents of $\mathrm{Al}_{2} \mathrm{O}_{3}$, $\mathrm{MgO}, \mathrm{MnO}$, and $\mathrm{TiO}_{2}$ are below detection limits excepting in few analyses. 

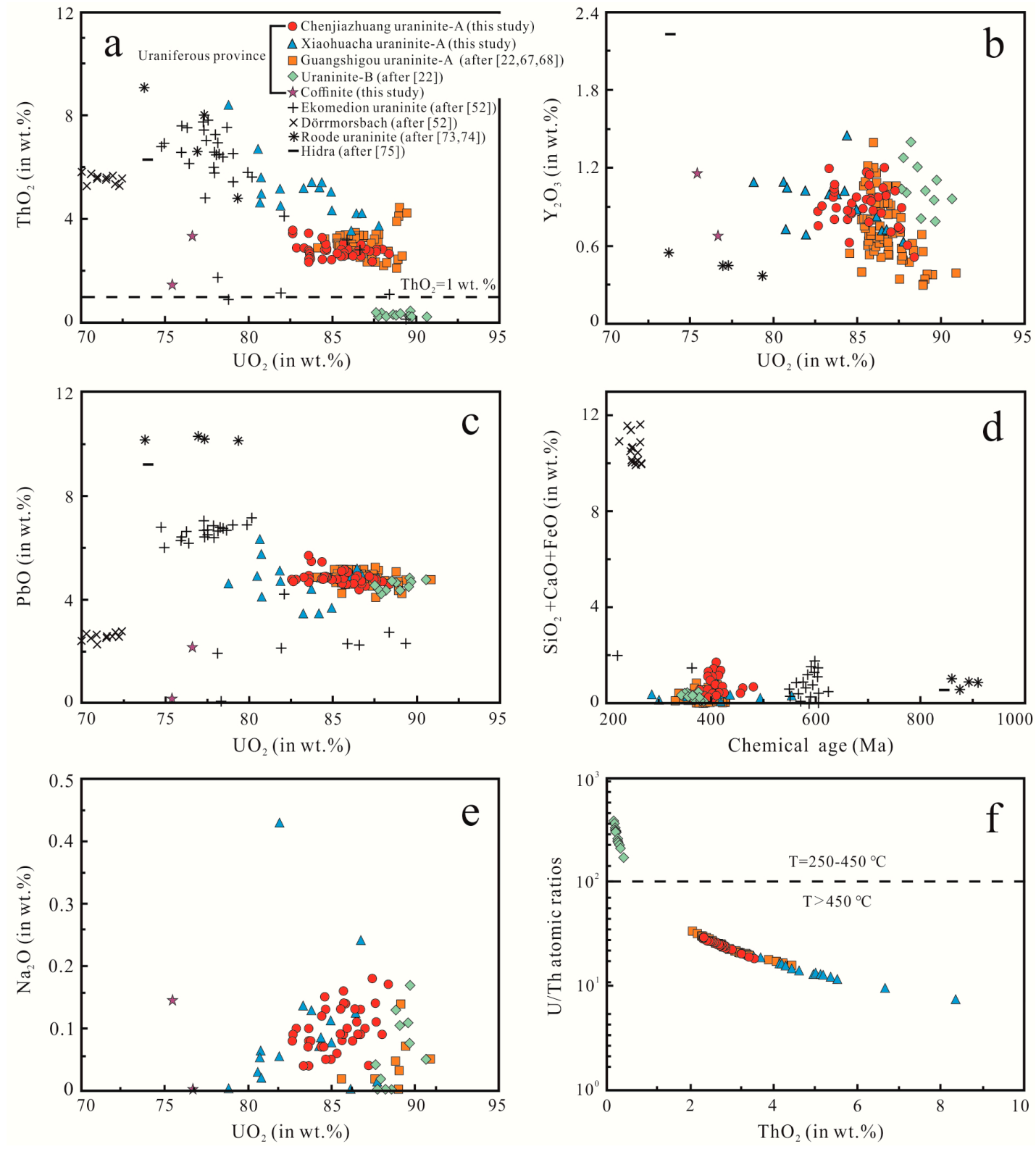

Figure 5. Binary diagrams of (a) $\mathrm{ThO}_{2}$ (wt.\%) vs. $\mathrm{UO}_{2}$ (wt.\%); (b) $\mathrm{Y}_{2} \mathrm{O}_{3}$ (wt.\%) vs. $\mathrm{UO}_{2}$ (wt.\%); (c) $\mathrm{PbO}$ (wt.\%) vs. $\mathrm{UO}_{2}$ (wt.\%); (d) $\sum \mathrm{SiO}_{2}+\mathrm{CaO}+\mathrm{FeO}$ (wt.\%) vs. chemical age (Ma); (e) $\mathrm{Na}_{2} \mathrm{O}$ (wt.\%) vs. $\mathrm{UO}_{2}$ (wt. $\%$ ); and (f) U/Th atomic ratio vs. $\mathrm{ThO}_{2}$ (wt.\%) showing chemical composition of the uraninite and coffinite.

Low total values (90.23-91.08 wt.\%) of coffinite result from a combination of partial oxidation of $\mathrm{U}^{4+}$ to $\mathrm{U}^{6+}$ and incorporation of constitution water into the crystal structure. Compositions of natural minerals and synthetic experiments suggest limited or complete solid solutions between coffinite, thorite, and xenotime end members [58,59]. Low Y levels (0.68-1.16 wt.\%, average $0.92 \pm 0.34$ wt.\%) in coffinite indicate a limited coffinite-xenotime solid solution, while coffinite incorporated $\mathrm{ThO}_{2}$ in abundances up to $3.36 \mathrm{wt}$.\%, suggesting that coffinite-thorite solid solution may be relevant for these uraniferous pegmatites. The coffinites also have higher $\mathrm{SiO}_{2}(5.34-7.74 \mathrm{wt}$ \% , average $6.54 \pm 1.7 \mathrm{wt} . \%)$, $\mathrm{CaO}(1.32-3.06$ wt.\%, average $2.19 \pm 1.23$ wt.\%), FeO (0.27-1.12 wt.\%, average $0.7 \pm 0.6$ wt. $\%)$, and $\mathrm{MgO}$ (0.13-1.18 wt.\%, average $0.16 \pm 0.04$ wt.\%), but lower $\mathrm{UO}_{2}(75.45-76.66$ wt. $\%$, average $70.06 \pm 0.86$ wt. $\%)$ and $\mathrm{PbO}(0.07-2.15$ wt. $\%$, average $1.11 \pm 1.47$ wt. \%) contents when compared with uraninite-A and uraninite-B (Figure 5). 


\subsection{Chemical Ages}

Estimation of the crystallization age of uraninite from chemical dating was presented in Table 2. Uraninite-A show calculated $\mathrm{U}-\mathrm{Th}-\mathrm{Pb}$ chemical age populations in the range of 360-427 Ma $(\mathrm{n}=42$, with 3 exceptions $>450 \mathrm{Ma}$ ) for the Chenjiazhuang uraninite-A, 286-438 Ma ( $\mathrm{n}=17$, exceptionally 496 and $555 \mathrm{Ma})$ for the Xiaohuacha uraninite-A, and 333-429 Ma ( $\mathrm{n}=90)$ for the Guangshigou uraninite-A. All of them exhibit main statistical peak ages calculated at ca. 400-420 Ma (Figure 6). By contrast, the uraninite- $\mathrm{B}$ show restricted and relatively lower calculated $\mathrm{U}-\mathrm{Th}-\mathrm{Pb}$ chemical ages ranging from 346-386 Ma ( $\mathrm{n}=11$, peak at 360-380 Ma, Figure 6).

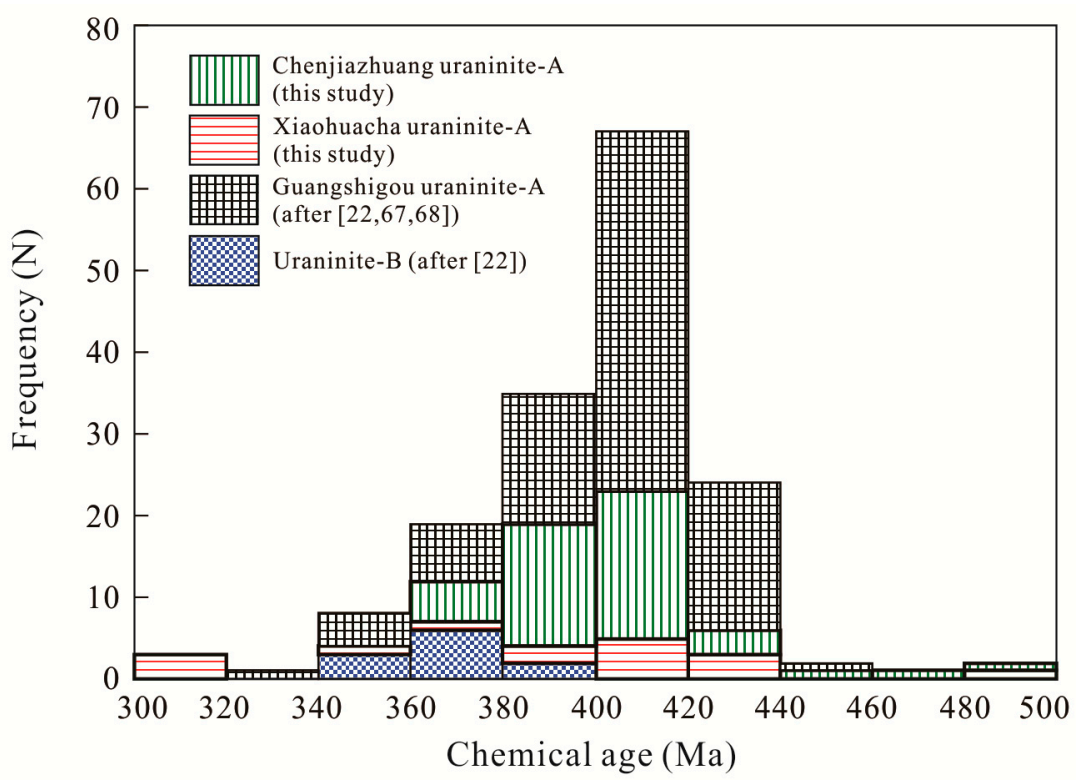

Figure 6. Histogram showing statistically age peaks for the uraninites from the Chenjiazhuang, Xiaohuacha, and Guangshigou biotite pegmatites in the uraniferous province.

\section{Discussion}

\subsection{Timing of the Uraninite (U) Mineralization}

Previous studies of geological characteristics, Sr-Nd-Hf-Pb isotopes, bulk-rock, and mineral chemistry have been conducted for the biotite pegmatites in the Shangdan uraniferous province (e.g., $[5,21,22,39])$. However, there is still controversy about the timing of $U$ mineralization. Many attempts to date these uraniferous pegmatites have replied on dating the ore-related accessory minerals. For instance, LA-ICP-MS zircon U-Pb dating gave weighted mean ${ }^{206} \mathrm{~Pb} /{ }^{238} \mathrm{U}$ ages of $414-417 \mathrm{Ma}$ [59], 414-415 Ma (unpub. data), and 413-415 Ma [22] for the Chenjiazhuang, Xiaohuacha, and Guangshigou biotite pegmatites, respectively; Liu [60] and Feng et al. [39] conducted biotite K-Ar dating of the Chenjiazhuang and Guangshigou biotite pegmatites, and obtained isotopic ages of 329.8-426.1 Ma and $400 \mathrm{Ma}$, respectively; Yuan et al. [5] and $\mathrm{Xu}$ and $\mathrm{Du}$ [45] reported a pyrite $\mathrm{Pb}-\mathrm{Pb}$ isochron age of $413 \pm 22 \mathrm{Ma}$ and two whole rock Rb-Sr isochron ages of 378 and $386 \mathrm{Ma}$ for the Guangshigou biotite pegmatite. The available ages are often inconsistent with each other, which may be caused by the large age uncertainty for some analyses, excess argon, or fluid-assisted resetting of the K-Ar and $\mathrm{Rb}-\mathrm{Sr}$ chronometers [61,62], resulting in uncertainties of interpretation. Besides this, petrographic evidence points to the formation of uraninite later than zircon (Figure 2f).

Directly dating of ore minerals is preferable to constrain the timing of mineralization [63]. Uraninite is prime target for directly dating of $U$ mineralization because it is common ore mineral in the pegmatite-hosted uraniferous province. Due to large difference in ionic radius and charge between $\mathrm{Pb}^{2+}(1.37 \AA)$ and $\mathrm{U}^{4+}(1.00 \AA)$ suggest incompatible $\mathrm{Pb}^{2+}$ crystal chemistry for uraninite [64]. Thus, 
$\mathrm{Pb}$ is typically excluded in primary growth, whereas varying amounts of radiogenic $\mathrm{Pb}$ produced by radioactive decay of $U$ can be retained in the crystal structure [8]. Combined with high $U$ abundance, it has been demonstrated that uraninite is an ideal proxy for dating $[17,19]$.

\subsubsection{Evaluation of Radioactive Pb-Loss}

Prior to the dating of $\mathrm{U}$ mineralization, it is necessary to evaluate the $\mathrm{Pb}$-loss of uraninite. There should be a negative correlation between $\mathrm{PbO}$ and $\mathrm{UO}_{2}$ in uraninite, which is attributed to closed-system radiogenic $\mathrm{Pb}$ production caused by decay of $\mathrm{U}$ [14]. However, such a trend is not observed as expected and some low-Pb uraninites appear (Figure $5 \mathrm{c}$ ), implying a release and loss of radioactive $\mathrm{Pb}$ to the matrix.

Based on rapid self-annealing kinetics, uraninite is known to a radiation-resistant phase [18]. Thus Pb-loss of uraninite (Figure 7) may be not caused by metamictization but can result from the following four processes: (I) removal of radiogenic $\mathrm{Pb}$ compensated by chemical substitutions of $\mathrm{Ca}$, $\mathrm{Si}$, and Fe during post-magmatic interactions with fluid [8,56], as evidenced by high sum of $\mathrm{Si}, \mathrm{Ca}$, and Fe for some analyses (>0.5 wt.\%) and by the presence of galena, pyrite, and calcite (Figure 2); (II) substitution of radiogenic $\mathrm{Pb}$ by water (magmatic fluid and late hydrothermal fluid, [19]); (III) hydrothermally-enhanced volume diffusion [65]; and (IV) replacement of uraninite by coffinite [9], as evidenced by the close association of uraninite and coffinite (Figure 2). Partially released Pb from uraninite, being not migrate long distances, are thought to have reprecipitated as secondary galena by sulfofication at high $f S_{2}$ (Figure $2 \mathrm{~d}, \mathrm{~g}$, [66]).

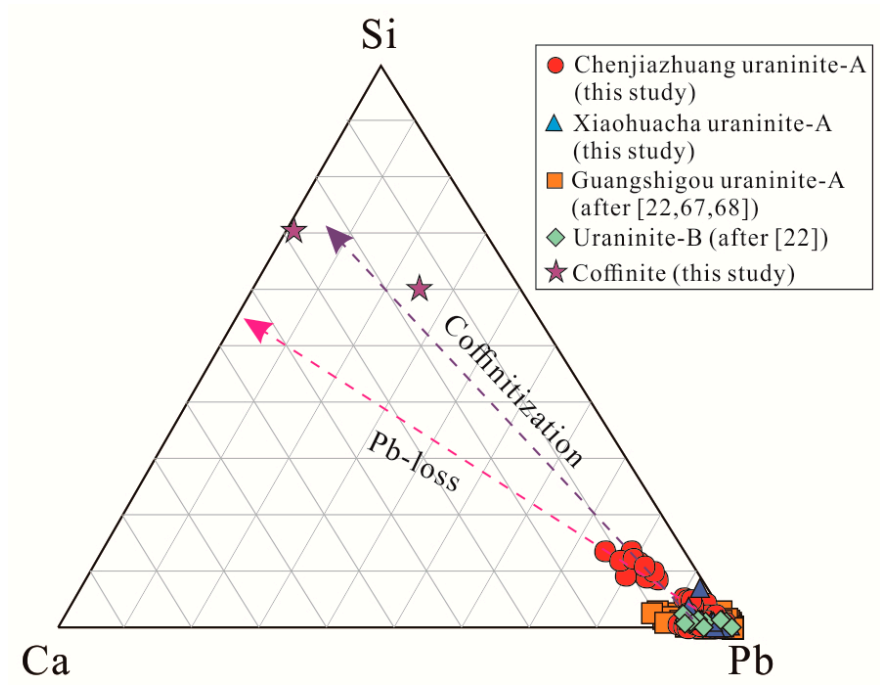

Figure 7. Ternary diagram of $\mathrm{Si}-\mathrm{Ca}-\mathrm{Pb}$ for uraninite and coffinite. Note that the two trends shown by the arrows indicate an overall $\mathrm{Pb}$-loss and $\mathrm{Ca}$ and $\mathrm{Si}$ gain during coffinitization.

\subsubsection{Timing of Uraninite (U) Mineralizing Event}

Our new age data for uraninite demonstrate that ID-TIMS U-Pb age (406.8 $\pm 0.5 \mathrm{Ma}$, Figure 4) for the Chenjiazhuang biotite pegmatite is good accordance with estimated chemical ages with a peak value of 400-420 Ma for the Chenjiazhuang, Xiaohuacha, and Guangshigou biotite pegmatites (Figure 6, [22,67,68]). These dating results are also identical within error to recent LA-ICP-MS uraninite $\mathrm{U}-\mathrm{Pb}$ analysis $(405 \pm 3 \mathrm{Ma})$ for the Guangshigou biotite pegmatites [69]. Although representative uraninite grain was carefully selected to conduct ID-TIMS U-Pb dating in this study, uraninite may have experienced local $\mathrm{Pb}$-loss, thus obtained ca. $407 \mathrm{Ma}$ can be considered as the lower limit of the timing of $\mathrm{U}$ mineralizing event. Combined with $\mathrm{U}-\mathrm{Pb}$ ages (ca. $415 \mathrm{Ma}$ ) of pre-crystallizing zircon (Figure 2f) obtained by Zhang et al. [59] and Yuan et al. [22], an Early Devonian age, constrained at 407-415 Ma, can be proposed for the timing of $U$ mineralization of these uraniferous pegmatites. 


\subsection{Significance of Composition and Comparison with Other Deposits}

Because of ionic radius of $\mathrm{Th}^{4+}(1.05 \AA)$ and $\mathrm{Y}^{3+}(1.019 \AA)$ being close to that of $\mathrm{U}^{4+}(1.00 \AA)$ in eightfold coordination [64], they can be easily incorporated into the uraninite structure, as shown by negative trends observed in the $\mathrm{ThO}_{2}$ vs. $\mathrm{UO}_{2}$ (Figure 5a) and $\mathrm{Y}_{2} \mathrm{O}_{3}$ vs. $\mathrm{UO}_{2}$ diagrams (Figure $5 \mathrm{~b}$ ), especially for the Xiaohuacha uraninite-A with Spearman rank correlation coefficients of -0.71 and -0.6 , respectively. Studies show that incorporation of Th into the uraninite structure is mainly a function of temperature and element availability, thus their $\mathrm{U} / \mathrm{Th}$ ratios can help in providing semi-quantitative estimation of crystallization temperature $[14,70,71]$. Uraninite with $\mathrm{U} / \mathrm{Th}$ ratio less than $10^{2}$ is commonly considered to be crystallized from high- $\mathrm{T}\left(>450{ }^{\circ} \mathrm{C}\right)$ magma or magmatic fluids, while uraninite formed at lower-T $\left(<250^{\circ} \mathrm{C}\right)$ diagenetic or hydrothermal fluids, characteristically contains high U/Th ratio (generally $>10^{3}$, $[10,13,52]$ ). Similar to pegmatite-related uraninites from Ekomédion (Cameroon), those from the Shangdan uraniferous province exhibit two distinct populations in terms of $\mathrm{ThO}_{2}$ contents (i.e. high $\mathrm{ThO}_{2}$ for uraninite-A and low $\mathrm{ThO}_{2}$ for uraninite-B, Figure 5a), corresponding to two distinct populations of crystallization temperature. The low $\mathrm{U} / \mathrm{Th}$ ratio $\left(<10^{2}\right)$ of uraninite-A is consistent with formation at high-T $\left(>450{ }^{\circ} \mathrm{C}\right)$ hydrous silicate melt (Figure $\left.5 \mathrm{f}\right)$. Based on the two-feldspar geothermometer, Feng [72] estimated ore-forming temperature $\left(582-607{ }^{\circ} \mathrm{C}\right)$ of the Guangshigou uraniferous pegmatites, similar to that $\left(600-650{ }^{\circ} \mathrm{C}\right.$, estimated by phase equilibria in the quartz-K-feldspar-albite- $\mathrm{H}_{2} \mathrm{O}$ system) of the Grenville U-Mo-REE pegmatites, Canada [2]. However, $\mathrm{ThO}_{2}$ contents of uraninite-A from the Shangdan uraniferous province are generally lower than those of uraninite from Dörrmorsbach (Germany), Roode and Hidra pegmatites (Norway) (Figure 5a, [52,73-75]), implying that uraninite-A crystallized at relatively lower temperature when compared to them. Based on uraninite-B with $\mathrm{U} / \mathrm{Th}$ ratios between $10^{2}$ and $10^{3}$, it may be tentatively inferred that uraninite-B formed at $250-450{ }^{\circ} \mathrm{C}$ (Figure 5f) similar to low-T uraninite in the Ekomedion pegmatites (Cameroon) [52]. In spite of relatively higher $\mathrm{ThO}_{2}$ contents for coffinite than uraninite-B, the condition favorable for coffinitization is generally low-T at $100-140{ }^{\circ} \mathrm{C}$ [8]. It is generally assumed that $Y$ contents in uraninite may also be controlled by formation temperature as Th and the crystal lattice permits substantial incorporation of $\mathrm{Y}$ at high- $\mathrm{T}$ (e.g., uraninite in Hidra pegmatites, Norway, [75]). However, we do not observe that uraninite- $\mathrm{B}$ has lower $\mathrm{Y}_{2} \mathrm{O}_{3}$ contents in contrast to uraninite-A as might be expect (Figure $5 b$ ). This may be caused by competition from pre- or co-crystallizing, potentially Y-incorporating garnet during the uraninite-A crystallization [52].

Based on incompatible behavior of $\mathrm{Pb}^{2+}$ and radiogenic $\mathrm{Pb}$ produced through time by radioactive decay, $\mathrm{Pb}$ contents in uraninite is a function of age [74]. The variability of $\mathrm{PbO}$ in uraninites from different pegmatites worldwide can be significant, ranging from ca. $2.5 \mathrm{wt} . \%$ in the Dörrmorsbach pegmatites (Germany) to $10 \mathrm{wt} . \%$ in the Roode pegmatites (Norway), with the pegmatite uraninite $(4.7 \mathrm{wt} . \%)$ from the Shangdan uraniferous province between them (Figure 5c), implying that they formed in different geological periods.

Elevated elemental impurities in uraninite is often expected as elemental substitutions (e.g., incorporation of $\mathrm{Ca}, \mathrm{Fe}$, and $\mathrm{Si}$ reaching several wt.\% through substitution with $\mathrm{Pb}$ ) during the post-crystallization alterations. A statistical treatment has been performed in order to reflect the correlation relationship among the major elements in uraninite (Table 3). We do not observe some negative correlations in the uraninite samples between $\mathrm{SiO}_{2}, \mathrm{CaO}, \mathrm{FeO}$ (or a combine of these elements), and $\mathrm{PbO}$, as might be expected (Figure $5 \mathrm{~d}$ ), with their absolute values of Spearman rank correlation coefficient $<0.5$ (Table 3). This phenomenon of no negative relationship with $\mathrm{PbO}$ also occurs other pegmatite-related deposits (e.g., Ekomedion in Cameroon, Roode in Norway, [52,73,74]). Alexandre et al. [74] argued that in addition to the degree of alteration, there are additional factors that control the mounts of these elements in uraninite. Due to ionic radius of Ca (1.06 $⿱$ ) being close to that of $\mathrm{U}(1.00 \AA)$, Ca can be integrated into the uraninite lattice though primary incorporation at the time of initial crystallization [19,52], such as fresh uraninites from the Dörrmorsbach pegmatite-related deposit (ca. 10.5 wt.\%, [52]) in Germany and Guérande granite-related hydrothermal deposit (as high as $9.55 \mathrm{wt} . \%$, [17]) in France. Previous studies have shown that elements incorporated during initial 
uraninite formation substituting for U (Th, Y, REE) also include Fe (eg. Cigar Lake unconformity-related deposit in Canada, ca. 0.2 wt.\%, [19]) and Si (e.g., Ike Tabular deposit in America, ca. 1.5 wt.\%, [74]). This may be confirmed by negative correlations between $\mathrm{UO}_{2}$ and $\mathrm{FeO}$ (correlation coefficient $=-0.58$ ) for the Xiaohuacha uraninite- $\mathrm{A}$, and $\mathrm{UO}_{2}$ and $\mathrm{SiO}_{2}(-0.69)$ for the Chenjiazhuang uraninite-A (Table 3). Accordingly, the incorporation of $\mathrm{Ca}, \mathrm{Fe}$, and $\mathrm{Si}$ into the uraninite lattice is controlled by both alteration degree and element availability.

\subsection{Episodic Hydrothermal Alterations of Uraninite}

Coffinite aggregates riming the uraninite (Figure $2 \mathrm{a}, \mathrm{j}-1$ ) and coffinite veinlets developed close to uraninite (Figure $2 \mathrm{j}, 1$ ) suggest nucleation on to the periphery of the uraninite grain or nearby (i.e., coffinitization). Both $\mathrm{U}^{4+}$ and $\mathrm{Th}^{4+}$ are soluble at high-T magmatic environments. Despite low solubility of $\mathrm{U}^{4+}$ in low-T fluids, $\mathrm{U}^{6+}$ is more soluble in under oxidizing conditions. However, by virtue of the low solubility of $\mathrm{Th}^{4+}$ (only valence state) in hydrothermal fluids, $\mathrm{Th}^{4+}$ is essentially immobile in hydrothermal environments $[13,76]$. Therefore, uraninites precipitated from hydrothermal fluid is depleted in $\mathrm{Th}^{4+}$, while those crystallized from magma or magmatic fluid is typically high in $\mathrm{Th}^{4+}$. Unlike uraninite-A with high $\mathrm{ThO}_{2}$ contents, uraninite-B contains much lower levels of $\mathrm{ThO}_{2}$ but similar $\mathrm{PbO}$ contents (Figure 5a,c). Accordingly, the integration of microtexture and chemical data of uranium minerals suggest that episodic hydrothermal alterations of primary magmatic uraninite-A are responsible for the formation of uraninite-B and coffinite.

An alteration mechanism involving coupled dissolution-reprecipitation reaction is suggested for the uraninite-B formation. The reaction was initiated by interaction between early magmatic uraninite-A and medium-T (250-450 $\left.{ }^{\circ} \mathrm{C}\right)$ hydrothermal fluid, resulting in local dissolution, decoupling of $U$ from $T h$, and in situ filling of dissolved regions by second uraninite-B containing little Th and elevated $U$ and $Y$. Micro-fractures permit the ingress of such a hydrothermal fluid into uraninite. The leached Th leaving the system may form thorite at $200-400{ }^{\circ} \mathrm{C}$ (cf. [10]). The petrographic evidence also supports a mechanism of dissolution of pre-existing uraninite and reprecipitation of secondary coffinite. Uranium released from uraninite does not appear to migrate over long distances but crystallize in the vicinity. The formation of coffinite is generally considered as related to a decrease of $f \mathrm{O}_{2}$ resulting from the interaction of soluble uranyl $\left(\mathrm{UO}_{2}\right)^{2+}$ ions in a Si-rich hydrothermal fluid with reducing agent (e.g., organic matter or sulfides, $[77,78])$. The fluid-induced leaching and dissolution of precursor uraninite have enriched the fluid in $U$. This process involves oxidative dissolution to more soluble $\left(\mathrm{UO}_{2}\right)^{2+}$ due to fluid radiolysis, release of excess oxygen during dissolution $[9,13]$, and increasing $f_{\mathrm{O} 2}$ through closed-system magma evolution. The close association of coffinite and calcite (Figure 2a,g,k) suggests that dissolved U was transported as stable carbonate complexes at low-T [79]. Note that the replacements of uraninite by coffinite were commonly observed at contacts with pyrite (Figure $2 \mathrm{~g}, \mathrm{k}, 1$ ), indicating that pyrite may act as reductant [76,80]. Reprecipitation of the necessary elements needed for coffinite under reducing, local disequilibrium (supersaturation) conditions favors the formation of coffinite near the pyrite $[6,9,56]$. Such a later $\mathrm{CO}_{2}$-rich fluid should also contain high silica activity to precipitate coffinite rather than uraninite [19]. A conversion from the uraninite to second coffinite is marked by an increase of $\mathrm{Si}$ and Ca coupled with a decrease of $\mathrm{U}$ and $\mathrm{Pb}$ (Figure 7).

\subsection{Uranium Circulation in the Uraniferous Province}

Uraninite and coffinite are the main carriers of $U$ in the Shangdan uraniferous province. Geochronological, petrographic, and microanalytical observations provide evidence for an interpretation of magmatic mineralization for the uraniferious pegmatites. Several lines of evidence in favor of formation of uraninite-B and coffinite are associated with late-stage hydrothermal U-events, including: (1) uranium minerals in close association (Figure 2), implying that coffinite and uraninite-B formed as a consequence of remobilization of $U$ from the magmatic uraninite- $A$; $(2)$ The presence of Th in low amounts of uraninite-B reflecting low crystallization temperature compared to uraninite-A (Figure 5f); (3) coffinitization occurring on the periphery of uraninite, pointing to late saturation of 
coffinite after uraninite; and (4) Coffinite with lower $\mathrm{PbO}$ contents compared to uraninite- $\mathrm{A}$ and uraninite-B, suggesting that coffinite with little radioactive decay postdates uraninite [10].

The crystallization sequence of $U$ minerals represents three discrete magmatic and hydrothermal events. High- $\mathrm{T}\left(450-607^{\circ} \mathrm{C}\right)$ precipitation of uraninite-A represents a primary $\mathrm{U}$ mineralizing event (ca. 407-415 Ma) associated with hydrous silicate melt, which can be interpreted as the first stage of $U$ circulation in the Shangdan uraniferous province. On the $\mathrm{U} / \mathrm{Th}$ vs. $\mathrm{ThO}_{2}$ binary diagram (Figure 5f), uraninite-A of the Chenjiazhuang, Xiaohuacha, and Guangshigou biotite pegmatites plots on a continuous trend, indicating a magmatic fractionation trend and precipitation at the magmatic stage. Of particular note is higher crystallization temperature for the Xiaohuacha uraninite-A (as indicated by lower U/Th ratios) than the Guangshigou and Chenjiazhuang uraninite-A, suggesting episodic injection and different emplacement depth of pegmatitic magma. The transformation of primary uraninite-A to secondary uraninite-B represents a hydrothermal event of $U$ remobilization. This process reflects that newly formed uraninite-B reprecipitated from in situ dissolution of the primary mineralization, resulting in the leaching of Th from the uraninite-A to hydrothermal fluids. Such a Th-rich fluid is responsible for the precipitation of thorite in the vicinity of uraninite (Figure 4i). Thus, during the medium- $\mathrm{T}\left(250-450^{\circ} \mathrm{C}\right)$ alteration in the Late Devonian, the local replacement of primary uraninite-A by secondary uraninite- $\mathrm{B}$, companied with thorite precipitation, could have been the second stage of $\mathrm{U}$ circulation. Coffinite reprecipitated as a later hydrothermal event of $U$ remobilization, involving the infiltration and dissolution of pre-existing uraninite by $\mathrm{CO}_{2}$ - and $\mathrm{SiO}_{2}$-rich fluids, and subsequent reprecipitation of the released $\mathrm{U}$ under reducing conditions. Accordingly, during the low-T $\left(100-140^{\circ} \mathrm{C}\right)$ alteration stage, the replacement of uraninite by secondary coffinite and formation of coffinite veinlets in association with calcite and pyrite indicate the third stage of $U$ circulation in the uraniferous province.

\section{Conclusions}

Integrating the study of petrographic observation, $\mathrm{U}$ mineral chemistry, ID-TIMS U-Pb dating, and chemical dating, together with previously published data, on the Chenjiazhuang, Xiaohuacha, and Guangshigou biotite pegmatites in the Shangdan uraniferous province lead us to the following conclusions:

(1) The ${ }^{206} \mathrm{~Pb} /{ }^{208} \mathrm{U}$ weighted mean age of $406.8 \pm 0.5 \mathrm{Ma}$ obtained by ID-TIMS is in line with the calculated U-Th- $\mathrm{Pb}$ chemical ages with prominent peaks at 400-420 Ma. Considering local Pb-loss for uraninite which is formed later than zircons, it can be inferred that the timing of $U$ mineralization is constrained in the Early Devonian (ca. 407-415 Ma).

(2) Based on microtextural relationship and chemical composition variation, three distinct generations of $\mathrm{U}$ minerals have been identified: uaninite-A (high Th-low $\mathrm{U}$-variable $\mathrm{Y}$ group), uranite- $\mathrm{B}$ (low Th-high $\mathrm{U}, \mathrm{Y}$ group), and coffinite (high $\mathrm{Si}$, Ca-low $\mathrm{U}, \mathrm{Pb}$ group).

(3) As the main magmatic carriers of $U$, uraninite-A display chemical characteristics typical of high-T $\left(450-607^{\circ} \mathrm{C}\right)$ magmatic uraninite, which were affected by two superimposed hydrothermal episodes. The local medium-T $\left(250-450^{\circ} \mathrm{C}\right)$ hydrothermal alteration involved the in situ replacement of primary uraninite-A by secondary uraninite- $\mathrm{B}$, companied with thorite precipitation. The low-T $\left(100-140^{\circ} \mathrm{C}\right)$ hydrothermal alteration involved the replacement of pre-existing uraninite by secondary coffinite and formation of coffinite veinlets in association with calcite and pyrite.

(4) The uraniferous province witnessed a three-stage circulation of $U$ in the pegmatitic system leading to primary mineralization-remobilization-reprecipitation: first, $U$ derived from the hydrous silicate melt was precipitated as primary uraninite-A, representing early-stage magmatic $U$ mineralization; then, $\mathrm{U}$ derived from the medium- $\mathrm{T}$ hydrothermal alteration of primary uraninite- $\mathrm{A}$ by U-Y-bearing fluid and coprecipitated with $Y$ to form second uraninite- $B$, representing a second-stage $U$ recycle by in situ dissolve and reprecipitation processes; finally, $\mathrm{U}$ derived from the low-T hydrothermal alteration of pre-existing uraninite by $\mathrm{CO}_{2}$ - and $\mathrm{SiO}_{2}$-rich fluids and interacted with pyrite, leading to a conversion of uraninite to secondary coffinite and representing a third-stage $U$ recycle by dissolve and reprecipitation processes. 
Author Contributions: Conceptualization, S.-Y.J. and J.L.; methodology, F.Y.; validation, F.Y.; formal analysis, F.Y.; investigation, J.L. and F.Y.; resources, Z.X. and X.H.; data curation, F.Y., S.Z., and G.L.; writing-original draft preparation, F.Y.; writing-review and editing, F.Y.; visualization, F.Y.; supervision, S.-Y.J. and J.L.; project administration, S.-Y.J. and J.L; funding acquisition, S.-Y.J. and J.L.

Funding: This study is supported by the National Key R\&D Program of China (No. 2017YFC0602405), the National Natural Science Foundation of China (No. 41730426, 41903043), China Postdoctoral Science Foundation (No. 2018M642948), the Fundamental Research Funds for the Central Universities (CUGCJ1709), the special fund from the State Key Laboratory of Geological Processes and Mineral Resources, China University of Geosciences (No. MSFGPMR03-2), and the work items of China Geological Survey (No. 12120114014401).

Conflicts of Interest: The authors declare no conflict of interest.

\section{References}

1. Linnen, R.L.; Lichtervelde, M.V.; Černý, P. Granitic pegmatites as sources of strategic metals. Elements 2012, 8, 275-280. [CrossRef]

2. Lentz, D. U, Mo, and REE mineralization in late-tectonic granitic pegmatites, southwestern Grenville Province, Canada. Ore Geol. Rev. 1996, 11, 197-227. [CrossRef]

3. Mckechnie, C.L.; Annesley, I.R.; Ansdell, K.M. Radioactive abyssal granitic pegmatites and l eucogranites in the Wollaston Domain, Northern Saskatchewan, Canada: Mineral compositions and conditions of emplacement in the Fraser Lakes area. Can. Mineral. 2012, 50, 1637-1667. [CrossRef]

4. Mckeough, M.A.; Lentz, D.R.; Mcfarlane, C.R.M.; Brown, J. Geology and evolution of pegmatite-hosted U-Th \pm REE-Y-Nb mineralization, Kulyk, Eagle, and Karin Lakes region, Wollaston Domain, northern Saskatchewan, Canada: Examples of the dual role of extreme fractionation and hybridization processes. J. Geosci. 2013, 58, 321-346. [CrossRef]

5. Yuan, F.; Liu, J.J.; Carranza, E.J.M.; Zhang, S.; Zhai, D.G.; Liu, G.; Wang, G.W.; Zhang, H.Y.; Sha, Y.Z.; Yang, S.S. Zircon trace element and isotopic ( $\mathrm{Sr}, \mathrm{Nd}, \mathrm{Hf}, \mathrm{Pb}$ ) effects of assimilation-fractional crystallization of pegmatite magma: A case study of the Guangshigou biotite pegmatites from the North Qinling Orogen, central China. Lithos 2017, 302-303, 20-36. [CrossRef]

6. Dahlkamp, F.J. Uranium Ore Deposits; Springer: Berlin, Germany, 1993; pp. 459-460.

7. Dill, H.G. Pegmatites and aplites: Their genetic and applied ore geology. Ore Geol. Rev. 2015, 69, 417-561. [CrossRef]

8. Finch, R.; Murakami, T. Systematics and paragenesis of uranium minerals. Rev. Mineral. Geochem. 1999, 38, 91-180.

9. Janeczek, J.; Ewing, R.C. Dissolution and alteration of uraninite under reducing conditions. J. Nucl. Mater. 1992, 190, 157-173. [CrossRef]

10. Macmillan, E.; Cook, N.J.; Ehrig, K.; Pring, A. Chemical and textural interpretation of late-stage coffinite and brannerite from the Olympic Dam IOCG-Ag-U deposit. Mineral. Mag. 2017, 81, 1323-1366. [CrossRef]

11. Alexandre, P.; Kyser, T.K. Effects of cationic substitutions and alteration in uraninite, and implications for the dating of uranium deposits. Can. Mineral. 2005, 43, 1005-1017. [CrossRef]

12. Eglinger, A.; André-Mayer, A.S.; Vanderhaeghe, O.; Mercadier, J.; Cuney, M.; Decrée, S.; Feybesse, J.L.; Milesi, J.P. Geochemical signatures of uranium oxides in the Lufilian belt: From unconformity-related to syn-metamorphic uranium deposits during the Pan-African orogenic cycle. Ore Geol. Rev. 2013, 54, 197-213. [CrossRef]

13. Hazen, R.M.; Ewing, R.C.; Sverjensky, D.A. Evolution of uranium and thorium minerals. Am. Mineral. 2009, 94, 1293-1311. [CrossRef]

14. Ozha, M.K.; Pal, D.C.; Mishra, B.; Desapati, T.; Shaji, T.S. Geochemistry and chemical dating of uraninite in the Samarkiya area, central Rajasthan, northwestern India-implication for geochemical and temporal evolution of uranium mineralization. Ore Geol. Rev. 2017, 88, 23-42. [CrossRef]

15. Kotzer, T.G.; Kyser, T.K. O, U, and Pb isotopic and chemical variations in uraninite: Implications for determining the temporal and fluid history of ancient terrains. Am. Mineral. 1993, 78, 1262-1274.

16. Cuney, M. Felsic magmatism and uranium deposits. Bull. Soc. Geol. Fr. 2014, 185, 75-92. [CrossRef] 
17. Ballouard, C.; Poujol, M.; Boulvais, P.; Mercadier, J.; Tartèse, R.; Venneman, T.; Deloule, E.; Jolivet, M.; Kéré, I.; Cathelineau, M.; et al. Magmatic and hydrothermal behavior of uranium in syntectonic leucogranites: The uranium mineralization associated with the Hercynian Guérande granite (Armorican Massif, France). Ore Geol. Rev. 2017, 80, 309-331. [CrossRef]

18. Macmillan, E.; Cook, N.J.; Ehrig, K.; Ciobanu, C.L.; Pring, A. Uraninite from the Olympic Dam IOCG-U-Ag deposit: Linking textural and compositional variation to temporal evolution. Am. Mineral. 2016, 101, 1295-1320. [CrossRef]

19. Martz, P.; Mercadier, J.; Perret, J.; Villeneuve, J.; Deloule, E.; Cathelineau, M.; Quirt, D.; Doney, A.; Ledru, P. Post-crystallization alteration of natural uraninites: Implications for dating, tracing, and nuclear forensics. Geochim. Cosmochim. Acta. 2019, 249, 138-159. [CrossRef]

20. Guo, X.F.; Szenknect, S.; Mesbah, A.; Labs, S.; Clavier, N.; Poinssot, C.; Ushakov, S.V.; Curtius, H.; Bosbach, D.; Ewing, R.C.; et al. Thermodynamics of formation of coffinite, $\mathrm{USiO}_{4}$. Proc. Natl. Acad. Sci. USA 2015, 112, 6551-6555. [CrossRef]

21. Chen, Y.W.; Bi, X.W.; Hu, R.Z.; Dong, S.H.; Cheng, D.J.; Feng, Z.S. Mineral chemistry of biotite and its implications for uranium mineralization in Guangshigou pegmatite type uranium deposit, South Shanxi province. Bull. Mineral. Petrol. Geochem. 2013, 33, 17-28. (In Chinese)

22. Yuan, F.; Liu, J.J.; Carranza, E.J.M.; Zhai, D.G.; Wang, Y.H.; Zhang, S.; Sha, Y.Z.; Liu, G.; Wu, J. The Guangshigou uranium deposit, northern Qinling Orogen, China: A product of assimilation-fractional crystallization of pegmatitic magma. Ore Geol. Rev. 2018, 99, 17-41. [CrossRef]

23. Meng, Q.R.; Zhang, G.W. Geologic framework and tectonic evolution of the Qinling orogen, central China. Tectonophysics 2000, 323, 183-196. [CrossRef]

24. Ratschbacher, L.; Hacker, B.R.; Calvert, A.; Webb, L.E.; Grimmer, J.C.; McWilliams, M.O.; Ireland, T.; Dong, S.W.; Hu, H.M. Tectonics of the Qinling (Central China): Tectonostratigraphy, geochronology, and deformation history. Tectonophysics 2003, 366, 1-53. [CrossRef]

25. Dong, Y.P.; Zhang, G.W.; Neubauer, F.; Liu, X.M.; Genser, J.; Hauzenbergerc, C. Tectonic evolution of the Qinling orogen, China: Review and synthesis. J. Asian Earth Sci. 2011, 41, 213-237. [CrossRef]

26. Yu, H.; Zhang, H.F.; Li, X.H.; Zhang, J.; Santosh, M.; Yang, Y.H.; Zhou, D.W. Tectonic evolution of the North Qinling Orogen from subduction to collision and exhumation: Evidence from zircons in metamorphic rocks of the Qinling Group. Gondwana Res. 2016, 30, 65-78. [CrossRef]

27. Liu, L.; Liao, X.Y.; Wang, Y.W.; Wang, C.; Santosh, M.; Yang, M.; Zhang, C.L.; Chen, D.L. Early Paleozoic tectonic evolution of the North Qinling Orogenic Belt in Central China: Insights on continental deep subduction and multiphase exhumation. Earth Sci. Rev. 2016, 159, 58-81. [CrossRef]

28. Wang, H.; Wu, Y.B.; Li, C.R.; Zhao, T.Y.; Qin, Z.W.; Zhu, L.Q.; Gao, S.; Zheng, J.P.; Liu, X.M.; Zhou, L.; et al. Recycling of sediment into the mantle source of K-rich mafic rocks: Sr-Nd-Hf-O isotopic evidence from the Fushui complex in the Qinling orogeny. Contrib. Mineral. Petrol. 2014, 168, 1062. [CrossRef]

29. Liu, B.X.; Qi, Y.; Wang, W.; Siebel, W.; Zhu, X.Y.; Nie, H.; He, J.F.; Chen, F.C. Zircon U-Pb ages and O-Nd isotopic composition of basement rocks in the North Qinling Terrain, central China: Evidence for provenance and evolution. Int. J. Earth Sci. 2013, 102, 2153-2173. [CrossRef]

30. Zhang, G.W.; Zhang, B.R.; Yuan, X.C.; Xiao, Q.H. The Qinling Orogenic Belt and Continental Dynamics; Science Press: Beijing, China, 2001. (In Chinese)

31. Liu, Q.; Wu, Y.B.; Wang, H.; Gao, S.; Qin, Z.W.; Liu, X.C.; Yang, S.H.; Gong, H.J. Zircon U-Pb ages and Hf isotope compositions of migmatites from the North Qinling terrane and their geological implications. J. Metamorph. Geol. 2014, 32, 177-193. [CrossRef]

32. Wu, Y.B.; Zheng, Y.F. Tectonic evolution of a composite collision orogen: An overview on the Qinling-Tongbai-Hong'an-Dabie-Sulu orogenic belt in central China. Gondwana Res. 2013, 23, 1402-1428. [CrossRef]

33. Song, S.G.; Su, L.; Li, X.H.; Niu, Y.L.; Zhang, L.F. Grenville-age orogenesis in the Qaidam-Qilian block: The link between South China and Tarim. Precambrian Res. 2012, 220-221, 9-22. [CrossRef]

34. Jiang, Y.H.; Jin, G.D.; Liao, S.Y.; Zhou, Q.; Zhao, P. Geochemical and Sr-Nd-Hf isotopic constraints on the origin of Late Triassic granitoids from the Qinling orogen, central China: Implications for a continental arc to continent-continent collision. Lithos 2010, 117, 183-197. [CrossRef] 
35. Diwu, C.R.; Sun, Y.; Zhao, Y.; Liu, B.X.; Lai, S.C. Geochronological, geochemical, and Nd-Hf isotopic studies of the Qinling complex, central China: Implications for the evolutionary history of the north Qinling Orogenic Belt. Geosci. Front. 2014, 5, 499-513. [CrossRef]

36. Shi, Y.; Yu, J.H.; Santosh, M. Tectonic evolution of the Qinling orogenic belt, Central China. New evidence from geochemical, zircon U-Pb geochronology and Hf isotopes. Precambrian Res. 2013, 231, 19-60. [CrossRef]

37. Yang, L.; Chen, F.K.; Yang, Y.Z.; Li, S.Q.; Zhu, X.Y. Zircon U-Pb ages of the Qinling Group in Danfeng area: Recording Mesoproterozoic and Neoproterozoic magmatism and Early Paleozoic metamorphism in the North Qinling terrain. Acta Geol. Sin. 2010, 26, 1589-1603. (In Chinese)

38. Lerch, M.F.; Xue, F.; Kröner, A. A Middle Silurian-Early Devonian magmatic arc in the Qinling Mountains of central China. J. Geol. 1995, 103, 437-449. [CrossRef]

39. Feng, M.Y.; Rong, J.S.; Sun, Z.F.; Xu, Z.Y.; Xie, H.J. Pegmatitic Uranium Deposit in the North Qinling; Atomic Energy Press: Beijing, China, 1996. (In Chinese)

40. Li, W.P.; Wang, T.; Wang, X.X.; Cao, H.Z. Single zircon age of the Huichizi composite body in North Qinling. Reg. Geol. China 2000, 19, 172-174. (In Chinese)

41. Qin, Z.W.; Wu, Y.B.; Siebel, W.; Gao, S.; Wang, H.; Abdallsamed, M.I.M.; Zhang, W.X.; Yang, S.H. Genesis of adakitic granitoids by partial melting of thickened lower crust and its implications for early crustal growth: A case study from the Huichizi pluton, Qinling orogen, central China. Lithos 2015, 238, 1-12. [CrossRef]

42. Wang, T.; Wang, X.X.; Tian, W.; Zhang, C.L.; Li, W.P.; Li, P. North Qinling Paleozoic granite associations and their variation in space and time: Implications for orogenicprocesses in the orogens of central China. Sci. China Ser. D Earth Sci. 2009, 39, 1359-1381. [CrossRef]

43. Zhang, C.L.; Liu, L.; Wang, T.; Wang, X.X.; Li, L.; Gong, Q.F.; Li, X.F. Granitic magmatism related to early Paleozoic continental collision in North Qinling. Chin. Sci. Bull. 2013, 58, 4405-4410. [CrossRef]

44. Wan, J.; Gao, L.B.; Wang, L.X. Metallogenic assessment environmental study and prospect of the granite-pegmatite-type uranium deposit in Shangxian-Danfeng triangle area, Shaanxi. Uranium Geol. 1992, 8, 258-263. (In Chinese)

45. Xu, Z.; Du, L.T. Characteristic and geneses of Rössing type uranium mineralization in Chenjiazhuang granite, Danfeng, Shanxi. Uranium Geol. 1988, 5, 257-265. (In Chinese)

46. Zhao, R.Y.; Li, W.H.; Jiang, C.Y.; Wang, C.Y.; Wang, J.B.; Wang, B.Y.; Hui, Z.P. The LA-ICP-MS zircon U-Pb dating, petro-geological characteristics of Hanglongmiao monzogranite in Danfeng area in eastern Qinling and their geological significance. Geol. Rev. 2014, 60, 1123-1130. (In Chinese)

47. Wang, X.X.; Wang, T.; Zhang, C.L. Neoproterozoic, Paleozoic, and Mesozoic granitoid magmatism in the Qinling Orogen, China. Constraints on orogenic process: J. Asian Earth Sci. 2013, 72, 129-151. [CrossRef]

48. Černý, P.; London, D.; Novák, M. Granitic pegmatites as reflections of their sources. Elements 2012, 8, $289-294$. [CrossRef]

49. Tu, J.R.; Xiao, Z.B.; Zhou, H.Y.; An, S.Q.; Li, G.Z.; Cui, Y.R.; Liu, W.G.; Li, H.M. U-Pb dating of single-grain uraninite by isotope dilution thermal ionization mass spectrometry. Ore Geol. Rev. 2019, 109, 407-412. [CrossRef]

50. Ludwig, K.R. User's Manual for Isoplot/Ex (version 2.2): A Geochronological Toolkit for Microsoft Excel. Berkely Geochronol. Cent. Spec. Publ. 2000, 1a, 1-55.

51. Kempe, U. Precise electron microprobe age determination in altered uraninite: Consequences on the intrusion age and the metallogenic significance of the Kirchberg granite (Erzgebirge, Germany). Contrib. Mineral. Petrol. 2003, 145, 107-118. [CrossRef]

52. Frimmel, H.E.; Schedel, S.; Brätz, H. Uraninite chemistry as forensic tool for provenance analysis. Appl. Geochem. 2014, 48, 104-121. [CrossRef]

53. Pal, D.C.; Rhede, D. Geochemistry and chemical dating of uraninite in the Jaduguda Uranium Deposit, Singhbhum Shear Zone, India-Implications for uranium mineralization and geochemical evolution of uraninite. Econ. Geol. 2013, 108, 1499-1515. [CrossRef]

54. Bowles, J.F.W. Age dating of individual grains of uraninite in rocks from electron microprobe analyses. Chem. Geol. 1990, 83, 47-53. [CrossRef]

55. Jaffey, A.H.; Flynn, K.F.; Glendenin, L.E.; Bentley, W.C.; Essling, A.M. Precision measurement of half-lives and specific activities of ${ }^{235} \mathrm{U}$ and ${ }^{238} \mathrm{U}$. Phys. Rev. C 1971, 4, 1889-1906. [CrossRef] 
56. Förster, H.J.; Rhede, D.; Stein, H.J.; Romer, R.L.; Tischendorf, G. Paired uraninite and molybdenite dating of the Knigshain granite: Implications for the onset of late-Variscan magmatism in the Lausitz Block. Int. J. Earth Sci. 2012, 101, 57-67. [CrossRef]

57. Xiong, Y.Q.; Shao, Y.J.; Zhou, H.D.; Wu, Q.H.; Liu, J.P.; Wei, H.T.; Zhao, R.C.; Cao, J.Y. Ore-forming mechanism of quartz-vein-type W-Sn deposits of the Xitian district in SE China: Implications from the trace element analysis of wolframite and investigation of fluid inclusions. Ore Geol. Rev. 2017, 83, 152-173. [CrossRef]

58. Förster, H.J. Composition and origin of intermediate solid solutions in the system thorite-xenotimezircon-coffinite. Lithos 2006, 88, 35-55. [CrossRef]

59. Zhang, S.; Liu, J.J.; Yuan, F.; Liu, G.; Wang, G.W.; Zhang, H.Y.; Zhang, H.Y. Zircon U-Pb geochronlogy, geochemistry of the granites and pegmatites and related uranium metallogenesis from the Chenjiazhuang deposit, Shaanxi Province. Earth Sci. Front. 2019, 24. in press (In Chinese)

60. Liu, D.C. Isotope geochronologic characteristics of uranium deposit from Chenjiazhuang. Mineral. Petrol. 1991, 43, 73-79. (In Chinese)

61. Jahn, B.M.; Liu, X.C.; Yui, T.F.; Morin, N.; Coz, B.L. High-pressure/ultrahigh-pressure eclogites from the Hong'an Block, East-Central China: Geochemical characterization, isotope disequilibrium and geochronological controversy. Contrib. Mineral. Petrol. 2005, 149, 499-526. [CrossRef]

62. Tartèse, R.; Ruffet, G.; Poujol, M.; Boulvais, P.; Ireland, T.R. Simultaneous resetting of the muscovite K-Ar and monazite U-Pb geochronometers: A story of fluids. Terra Nova 2011, 23, 390-398. [CrossRef]

63. Yuan, S.D.; Peng, J.T.; Hao, S.; Li, H.M.; Geng, J.Z.; Zhang, D.L. In situ LA-MC-ICP-MS and ID-TIMS U-Pb geochronology of cassiterite in the giant Furong tin deposit, Hunan Province, South China: New constraints on the timing of tin-polymetallic mineralization. Ore Geol. Rev. 2011, 43, 235-242. [CrossRef]

64. Shannon, R.D. Revised effective ionic radii and systematic studies of interatomic distances in halides and chalcogenides. Acta Crystallogr. 1976, 32, 751-767. [CrossRef]

65. Brookins, D.G. Radionuclide behavior at the Oklo nuclear reactor, Gabon. Waste Manag. 1990, 10, $285-296$. [CrossRef]

66. Lumpkinm, G.R.; Chakoumakos, B.C. Chemistry and radiation effects of thorite-group minerals from the Harding pegmatite, Taos County, New Mexico. Am. Mineral. 1988, 73, 1405-1419. [CrossRef]

67. Ge, X.K. Electron Probe Chemical Dating Development and Its Application in Uranium and U-Bearing Mineral Research. Ph.D. Thesis, Bejing Research Institute of Uranium Geology, Bejing, China, 2013. (In Chinese)

68. Guo, G.L.; Zhang, Z.S.; Liu, X.D.; Feng, Z.S.; Lai, D.R.; Zhou, W.R. EPMA chemical U-Th-Pb dating of uraninite in guangshigou uranium deposit. J. East China Inst. Technol. 2012, 35, 309-314. (In Chinese)

69. Wu, Y.; Qin, M.K.; Guo, D.F.; Fan, G.; Liu, Z.Y.; Guo, G.L. The Latest In-Situ uraninite U-Pb age of the Guangshigou uranium deposit, Northern Qinling Orogen, China: Constraint on the Metallogenic Mechanism. Acta Geol. Sin. 2018, 92, 389-391.

70. Balboni, E.; Jones, N.; Spano, T.; Simonetti, A.; Burns, P.C. Chemical and Sr isotopic characterization of North America uranium ores: Nuclear forensic applications. Appl. Geochem. 2016, 74, 24-32. [CrossRef]

71. Depiné, M.; Frimmelm, H.E.; Emsbo, P.; Koenig, A.E.; Kern, M. Trace element distribution in uraninite from Mesoarchaean Witwatersrand conglomerates (South Africa) supports placer model and magmatogenic source. Miner. Depos. 2013, 48, 423-435. [CrossRef]

72. Feng, Z.S. The preliminary research on feldspar of Guangshigou pegmatite-typeuranium deposit, south Shanxi province. Geol. Chem. Miner. 2012, 34, 71-76. (In Chinese)

73. Alexandre, P.; Peterson, R.; Joy, B. Sector zoning in uraninite. Can. Mineral. 2015, 53, 693-703. [CrossRef]

74. Alexandre, P.; Kyser, K.; Layton-Matthews, D.; Joy, B. Chemical compositions of natural uraninite. Can. Mineral. 2016, 53, 1-30. [CrossRef]

75. Hetherington, C.J.; Harlov, D.E. Metasomatic thorite and uraninite inclusions in xenotime and monazite from granitic pegmatites, Hidra anorthosite massif, southwestern Norway: Mechanics and fluid chemistry. Am. Mineral. 2013, 93, 806-820. [CrossRef]

76. Göb, S.; Guhring, J.E.; Bau, M.; Markl, G. Remobilization of U and REE and the formation of secondary minerals in oxidized U deposits. Am. Mineral. 2013, 98, 530-548. [CrossRef]

77. Deditius, A.P.; Utsunomiya, S.; Wall, M.A.; Pointeau, V.; Ewing, R.C. Crystal chemistry and radiation-induced amorphization of P-coffinite from the natural fission reactor at Bangombé, Gabon. Am. Mineral. 2009, 94, 827-837. [CrossRef] 
78. Costin, D.T.; Mesbah, A.; Clavier, N.; Dacheux, N.; Poinssot, D.; Szenknect, S.; Ravaux, J. How to explain the difficulties in the coffinite synthesis from the study of uranothorite? Inorg. Chem. 2011, 50, 11117-11126. [CrossRef] [PubMed]

79. Hu, R.Z.; Bi, X.W.; Zhou, M.F.; Peng, J.T.; Su, W.C.; Liu, S.; Qi, H.W. Uranium metallogenesis in South China and its relationship to crustal extension during the Cretaceous to Tertiary. Econ. Geol. 2008, 103, 583-598. [CrossRef]

80. Fayek, M.; Janeczek, J.; Ewing, R.C. Mineral chemistry and oxygen isotopic analyses of uraninite, pitchblende and uranium alteration minerals from the Cigar Lake deposit, Saskatchewan, Canada. Appl. Geochem. 1997, 12, 549-565. [CrossRef]

(C) 2019 by the authors. Licensee MDPI, Basel, Switzerland. This article is an open access article distributed under the terms and conditions of the Creative Commons Attribution (CC BY) license (http://creativecommons.org/licenses/by/4.0/). 